\title{
Millimeter and some near infra-red observations of short-period Miras and other AGB stars ${ }^{\star}$
}

\author{
M.A.T. Groenewegen ${ }^{1}$, F. Baas ${ }^{2}$, J.A.D.L. Blommaert ${ }^{3}$, R. Stehle ${ }^{4}$, E. Josselin ${ }^{5}$, and R.P.J. Tilanus ${ }^{2}$ \\ 1 Max-Planck Institut für Astrophysik, Karl-Schwarzschild-Straße 1, D-85740 Garching, Germany \\ 2 Joint Astronomy Centre, 660 N. A'ohōkū Place, University Park, Hilo, HI 96720, U.S.A. \\ 3 ISO Data Centre, Astrophysics Division of ESA, P.O. Box 50727, E-28080 Villafranca-Madrid, Spain \\ ${ }^{4}$ Department of Physics \& Astronomy, University of Leicester, University Road, Leicester LE1 7RH, UK \\ 5 Observatorio Astronómico Nacional (I.G.N.), Apartado 1143, E-28801 Alcalá de Henares, Spain
}

Received May 10; accepted September 8, 1999

\begin{abstract}
Millimeter observations of 48 oxygen- and 20 carbon-rich AGB Miras with periods shorter than 400 days are presented. In addition, observations of 14 O-rich and $15 \mathrm{C}$-stars with longer, or no known, periods have also been obtained.

The detection statistics is as follows: in ${ }^{12} \mathrm{CO} J=1-0$ and 2-1 we observed 97 stars, and detected 66 in at least one line. We find 24 new detections in the 1-0 line, 38 new detections in the 2-1 line, and 29 stars have been detected for the first time in one or both lines. In ${ }^{12} \mathrm{CO} J=3-2$ we observed 14 stars and detected 11, with 4 new detections. In ${ }^{13} \mathrm{CO} J=2-1,3-2$ we observed 2 stars and had one new detection. In $\mathrm{HCN}(1-0)$ we observed 5 carbon stars and detected 3 , one new. In $\mathrm{SO}\left(6_{5}-5_{4}\right)$ we observed the same 5 stars and detected none. In $\mathrm{CS}(3-2)$ we observed 8 carbon stars and detected 3 , all new. In $\mathrm{SiO}(3-2$, $v=0$ ) we observed 34 O-rich stars and detected 25, all new except one.
\end{abstract}

Near-infrared $J H K$ photometry is presented for seven stars. For four stars it is the first NIR data published.

The luminosity and dust mass loss rate are obtained for seven very red stars with unknown pulsation period from modelling the spectral energy distribution (SED) and IRAS LRS spectra. Thereby, a new IR supergiant is

Send offprint requests to: M. Groenewegen

e-mail: groen@mpa-garching.mpg.de

* The James Clerk Maxwell Telescope is operated by the Observatories on behalf of the Science and Engineering Council of the UK, the Netherlands Organization for Scientific Research, and the National Research Council of Canada. Based on observations made with the Carlos Sanchez telescope operated on the island of Tenerife in the Spanish Observatorio del Teide of the Instituto de Astrofísica de Canarias. Based on observations collected at the European Southern Observatory, La Silla, Chile within programs ESO 57.E-0105, 59.E-0198 and 61.E-0254. Based on data from the ESA Hipparcos astrometry satellite. confirmed (AFGL 2968). For the rest of the sample, luminosity and distance are obtained in a variety of ways: using HIPPARCOS parallaxes, period-luminosity and period- $M_{K}$-relations combined with apparent $K$ magnitudes, and kinematic distances.

The dust mass loss rate is obtained from model fitting of the SED (either from the literature, or presented in the present paper), or from the observed IRAS $60 \mu \mathrm{m}$ flux, corrected for the photospheric contribution. The gas mass loss rate is derived from the observed CO line intensities, as presented here, combined with existing literature data, if any. This allows the derivation of the dust-to-gas ratio. Our and literature CO $J=3-2$ data has been used to calibrate the relation between mass loss rate and peak intensity of the $\mathrm{CO}(3-2)$ line.

Diagrams showing mass loss rate, dust-to-gas ratio and expansion velocity versus pulsation period are presented. Our observations confirm the existence of an upper limit for the expansion velocity of $\mathrm{C}$ - and O-rich stars, and that this maximum is larger for C-stars, as predicted by the theory of radiation pressure on dust particles.

Key words: circumstellar matter — stars: mass loss stars: AGB

\section{Introduction}

Previous studies have shown that mass loss rates increase with increasing pulsation periods in Miras. For example, Whitelock (1990) and Whitelock et al. (1994) derived the mass loss rates for oxygen-rich Miras in the Galactic Bulge and the South Galactic Cap, respectively, and found this 
trend. The mass loss rates were based on dust emission and then converted to a total mass loss rate based on a constant dust-to-gas ratio and expansion velocity for all stars in their respective samples.

In a different approach to derive the dust mass loss rate, Groenewegen et al. (1998) fitted the Spectral Energy Distributions (SEDs) and mid-infrared IRAS LRS spectra of 44 carbon Mira variables with a dust radiative transfer model. In that paper the total mass loss rate was then derived from a modified relation between the photon momentum transfer rate $(L / c)$ and the momentum transfer rate of the wind $\left(\dot{M} v_{\infty}\right)$.

There is an alternative method to derive the total mass loss rate and that is through molecular emission lines, notably CO which is the most abundant molecule after $\mathrm{H}_{2}$. Many $\mathrm{CO}$ observations have been published for AGB stars over the past years (see Loup et al. 1993 for a compilation up to that epoch; more recent work include Bieging \& Latter 1994; Sahai \& Liechti 1995; Young 1995; Groenewegen et al. 1996; Knapp et al. 1998; Olofsson et al. 1998; Neri et al. 1998; Kerschbaum \& Olofsson 1998; Groenewegen \& de Jong 1998). CO observations are the main focus here as well. As the results in Groenewegen et al. (1998) suggested that the overall good relation between mass loss rate and pulsation period might break down for periods shorter than about 400 days we concentrated on the shorter period range, and also included both O- and C-rich Miras. This possible breakdown of a mass loss-period relation might be related to the fact that radiation pressure on dust is no longer sufficient to drive the mass loss at lower luminosity (Dominik et al. 1990).

The paper is organised as follows. In Sect. 2 the sample of stars is introduced and the millimeter observations are described. In Sect. 3 the line profiles and observational results are presented. In Sect. 4 the infrared observations are described. In Sect. 5 the analysis is performed, which includes the modelling of the SEDs and LRS spectra for 7 stars, estimation of luminosity and distance using several methods, derivation of the dust- and gas-mass loss rate. In Sect. 6 we comment on the various correlations between mass loss rate and pulsation period, expansion velocity, etc.

\section{The observations}

Observations were performed with the IRAM-30 m-telescope and the SEST each on three different occasions and with the JCMT on one occasion. In Table 1 we list the observations of the different runs, the observed coordinates, chemical type $(\mathrm{C}$ or $\mathrm{M})$ and pulsation period. In addition, references are given to previous detections in $\mathrm{CO}$ and in the case of non-detections (previously and by us) we refer to radial velocities obtained by other means, if avaialable. The stellar coordinates are in most cases taken from the SIMBAD data base (when listed to $\lesssim 0.2^{\prime \prime}$ accuracy), the Hipparcos input catalog, the Stephenson (1989) catalog of carbon stars, or derived from the Digitised Sky Survey. In few cases the IRAS coordinates are used, mostly for the less known carbon stars and the stars listed under their IRAS name. The pulsation periods are taken from the 4th edition of the General Catalog of Variable Stars (GCVS, Kholopov et al. 1985), Jones et al. (1990), Le Bertre (1992) and Joyce et al. in preparation.

The data obtained with the IRAM-30 m-telescope were taken in the period between 23 and 29 December 1994, between 26 and 28 January 1995 and on the 3rd and 4th of August 1995. For the December 1994 run (observers MG and JB) the ${ }^{12} \mathrm{CO} J=1-0$ and $J=2-1$ lines, and for some stars also the $\mathrm{HCN}(1-0)$ and $\mathrm{SO}\left(6_{5}-5_{4}\right)$ lines, were observed simultaneously using the $230 \mathrm{GHz}$ and $3 \mathrm{~mm}$ SIS receivers. Both the two $1 \mathrm{MHz}$ filter banks and the autocorrelator were used as backends. In cases where both data sets are available with good $S / N$ we only present the one with the highest frequency resolution. The same procedure is adopted for the other observations discussed below. Table 3 gives the channel spacing which we finally decided to use.

The targets were observed using wobbler switching during all runs with the IRAM-30 m-telescope, mostly with a throw of $120^{\prime \prime}$ in azimuth.

Baselines were removed and all temperatures are on a main-beam brightness scale (the same applies for all temperatures presented in this paper). Due to different definitions used at different telescopes the observed antenna temperatures $\left(T_{\mathrm{A}}\right)$ are converted to main-beam brightness temperatures $\left(T_{\mathrm{mb}}\right)$ by using $T_{\mathrm{mb}}=T_{\mathrm{A}} / B_{\text {eff }}$ for the SEST and JCMT data (discussed below), and $T_{\mathrm{mb}}=T_{\mathrm{A}} \times F_{\text {eff }} / B_{\text {eff }}$ for the IRAM data, where $F_{\text {eff }}$ and $B_{\text {eff }}$ are respectively the forward and main-beam efficiencies (listed in Table 2 together with the FWHM beam widths).

The comparison with the calibration sources (supplemented by consistency checks from stars observed during more days, or even observed both in the December and January runs, as well as published results obtained with the IRAM-30 m-telescope) indicated that the CO $J=1-0$ and the HCN observations during the December 1994 run are consistent with the calibration observations. The situation is different for the CO $J=2-1$ and SO data. The observed $\mathrm{CO} J=2-1$ data had to be multiplied by factors $1.1-1.5$ to obtain agreement with previous results. For the SO transitions there are no calibration sources and only very few published data. The $230 \mathrm{GHz}$ receiver was tuned to the SO line during two consecutive days during the December 1994 run. We observed IRC +10011 on both days and compared our data to that of Bujarrabal et al. (1994). On the first day our brightness temperature scale was too low by a factor of 2.2 ; on the second day we found agreement between the two. By scaling the result for VY CMa obtained with the 
Table 1. The sample

\begin{tabular}{|c|c|c|c|c|c|}
\hline Name & $\begin{array}{l}\text { R.A. } \\
(1950)\end{array}$ & $\begin{array}{l}\text { Dec. } \\
(1950)\end{array}$ & Type & $\begin{array}{l}\text { Period } \\
\text { (days) }\end{array}$ & References \\
\hline \multicolumn{6}{|c|}{ IRAM December 1994 run } \\
\hline V390 Cas & 012516.3 & +620109 & $\mathrm{C}$ & $362^{a}$ & \\
\hline PT Cas & 013451.5 & +554256 & $\mathrm{C}$ & 300 & \\
\hline CN Per & 020312.7 & +563643 & $\mathrm{C}$ & 384 & \\
\hline R For & 022701.31 & -261914.8 & $\mathrm{C}$ & 388.7 & Loup et al. (1993), Olofsson et al. (1993, 1998) \\
\hline AFGL 5076 & 023434.6 & +542219 & $\mathrm{C}$ & - & Volk et al. (1993) \\
\hline IRAS $02408+5458$ & 024049.1 & +545835 & $\mathrm{C}$ & - & \\
\hline GY Per & 042550.76 & +392711.2 & $\mathrm{C}$ & 377.8 & \\
\hline UV Aur & 051833.31 & +322751.4 & $\mathrm{C}$ & $394.2^{b}$ & SIMBAD \\
\hline ZZ Gem & 062056.5 & +250331 & $\mathrm{C}$ & 317.0 & \\
\hline AFGL 935 & 062304.64 & -093020.1 & $\mathrm{C}$ & 494 & Loup et al. (1993) \\
\hline IRAS $06582+1507$ & 065817.3 & +150758 & $\mathrm{C}$ & - & Loup et al. (1993), Volk et al. (1993) \\
\hline $\mathrm{R} \mathrm{CMi}$ & 070557.56 & +100616.1 & $\mathrm{C}$ & 337.8 & SIMBAD \\
\hline VX Gem & 070958.6 & +144111 & $\mathrm{C}$ & 379.4 & SIMBAD \\
\hline C1698 & 072209.3 & -043158 & $\mathrm{C}$ & - & Engels (1994) \\
\hline R Pyx & 084323.8 & -280104 & $\mathrm{C}$ & 364.7 & \\
\hline $\mathrm{V} \mathrm{CrB}$ & 154743.89 & +394320.5 & $\mathrm{C}$ & 357.6 & $\begin{array}{l}\text { Loup et al. (1993), Olofsson et al. (1993), } \\
\text { Knapp et al. (1998), Neri et al. (1998) }\end{array}$ \\
\hline $\mathrm{V} O \mathrm{Oph}$ & 162356.58 & -121854.7 & $\mathrm{C}$ & 297.2 & \\
\hline AFGL 2310 & 190053.05 & +072621.9 & $\mathrm{C}$ & 577 & Loup et al. (1993), Likkel \& Miao (1996) \\
\hline AFGL 2368 & 191735.39 & -080751.0 & $\mathrm{C}$ & 676 & Loup et al. (1993) \\
\hline AFGL 2686 & 205700.28 & +271451.4 & $\mathrm{C}$ & 750 & Loup et al. (1993) \\
\hline V1549 Cyg & 210332.6 & +513618 & $\mathrm{C}$ & 533 & Loup et al. (1993) \\
\hline AX Cep & 212615.0 & +700016 & $\mathrm{C}$ & 395.0 & \\
\hline AFGL 5625 & 213150.1 & +563113 & $\mathrm{C}$ & - & Loup et al. (1993), Volk et al. (1993), Neri et al. (1998) \\
\hline CIT 13 & 213206.06 & +385052.9 & $\mathrm{C}$ & 470 & Loup et al. (1993), Olofsson et al. (1993) \\
\hline $\mathrm{AO} \mathrm{Lac}$ & 224035.9 & +511536 & $\mathrm{C}$ & 300 & \\
\hline LS Cas & 233545.8 & +554512 & $\mathrm{C}$ & 340 & \\
\hline AFGL 230 & 013027.63 & +621131.2 & $\mathrm{O}$ & 1525 & Loup et al. (1993), Neri et al. (1998) \\
\hline AFGL 5093 & 032041.48 & +652132.8 & $\mathrm{O}$ & 1276 & Loup et al. (1993) \\
\hline AFGL 5097 & 032923.65 & +601004.4 & $\mathrm{O}$ & - & Loup et al. (1993) \\
\hline NV Aur & 050719.68 & +524853.9 & $\mathrm{O}$ & 635 & Loup et al. (1993) \\
\hline AFGL 712 & 051306.6 & +453048 & $\mathrm{O}$ & 1088 & \\
\hline IRAS $05284+1945$ & 052827.2 & +194508 & $\mathrm{O}$ & - & \\
\hline IRAS $06403-0138$ & 064019.1 & -013805 & $\mathrm{O}$ & - & \\
\hline IRAS $07113-2747$ & 071123.1 & -274746 & $\mathrm{O}$ & - & \\
\hline $\mathrm{OH} 10.1-0.1$ & 180517.95 & -201641.0 & $\mathrm{O}$ & - & Blommaert et al. (1994) \\
\hline IRAS $21554+6204$ & 215529.6 & +620424 & $\mathrm{O}$ & - & Loup et al. (1993) \\
\hline AFGL 2885 & $22 \quad 1742.7$ & +593617 & $\mathrm{O}$ & 1570 & Loup et al. (1993) \\
\hline AFGL 2968 & 224800.9 & +600201 & $\mathrm{O}$ & - & Josselin et al. (1998) \\
\hline \multicolumn{6}{|c|}{ IRAM January 1995 run } \\
\hline Y Per & 032418.07 & +440012.6 & $\mathrm{C}$ & 248.6 & \\
\hline R Ori & 045617.62 & +080319.7 & $\mathrm{C}$ & 377.1 & \\
\hline V Aur & 062015.4 & +474403 & $\mathrm{C}$ & 353.0 & \\
\hline ZZ Gem & 062056.5 & +250331 & $\mathrm{C}$ & 317.0 & \\
\hline $\mathrm{R} \mathrm{CMi}$ & 070557.56 & +100616.1 & $\mathrm{C}$ & 337.8 & \\
\hline R Pyx & 084323.8 & -280104 & $\mathrm{C}$ & 364.7 & \\
\hline $\mathrm{V}$ CrB & 154743.89 & +394320.5 & $\mathrm{C}$ & 357.6 & see above \\
\hline $\mathrm{V} \mathrm{Oph}$ & 162356.58 & -121854.7 & $\mathrm{C}$ & 297.2 & \\
\hline AX Cep & 212615.0 & +700016 & $\mathrm{C}$ & 395.0 & \\
\hline U Ori & 055250.95 & +201008.1 & $\mathrm{O}$ & 368.3 & Young (1995), Knapp et al. (1998) \\
\hline R LMi & 094234.75 & +344433.9 & $\mathrm{O}$ & 372.2 & Loup et al. (1993), Young (1995) \\
\hline R Leo & 094452.22 & +113942.0 & $\mathrm{O}$ & 310.0 & $\begin{array}{l}\text { Loup et al. (1993), Bieging \& Latter (1994), } \\
\text { Young (1995), Stanek et al. (1995), Olofsson et al. (1998) }\end{array}$ \\
\hline RS Vir & 142446.00 & +045406.3 & $\mathrm{O}$ & 354.0 & \\
\hline R Ser & 154823.26 & +151703.1 & $\mathrm{O}$ & 356.4 & Loup et al. (1993), Young (1995), Knapp et al. (1998) \\
\hline $\mathrm{X} \mathrm{Oph}$ & 183557.57 & +084719.9 & $\mathrm{O}$ & 328.9 & Young (1995) \\
\hline R Peg & 230408.27 & +101622.8 & $\mathrm{O}$ & 378.1 & Young (1995) \\
\hline \multicolumn{6}{|c|}{ IRAM August 1995 run } \\
\hline R Cet & 022328.71 & -002411.0 & $\overline{\mathrm{O}}$ & 166.2 & \\
\hline $\mathrm{R}$ Tri & 023400.02 & +340251.6 & $\mathrm{O}$ & 266.9 & SIMBAD \\
\hline R Tau & 042533.46 & +100308.9 & $\mathrm{O}$ & 320.9 & \\
\hline T Lep & 050243.20 & -215818.7 & $\mathrm{O}$ & 368.1 & \\
\hline X Hya & 093306.94 & -142803.5 & $\mathrm{O}$ & 301.1 & Young (1995) \\
\hline $\mathrm{R}$ Aqr & 234114.18 & -153341.8 & $\mathrm{O}$ & 387.0 & \\
\hline \multicolumn{6}{|c|}{ JCMT August 1995 run } \\
\hline R For & 022701.31 & -261914.8 & $\overline{\mathrm{C}}$ & 388.7 & see above \\
\hline UV Aur & 051833.31 & +322751.4 & $\mathrm{C}$ & 394.2 & see above \\
\hline
\end{tabular}


Table 1. continued

\begin{tabular}{|c|c|c|c|c|c|}
\hline Name & $\begin{array}{l}\text { R.A. } \\
(1950) \\
\end{array}$ & $\begin{array}{l}\text { Dec. } \\
(1950) \\
\end{array}$ & Type & $\begin{array}{l}\begin{array}{l}\text { Period } \\
\text { (days) }\end{array} \\
\end{array}$ & References \\
\hline R Cap & 200830.18 & -142502.3 & $\mathrm{C}$ & 345.1 & \\
\hline AX Cep & 212615.0 & +700016 & $\mathrm{C}$ & 395.0 & \\
\hline $\mathrm{S} \mathrm{Scl}$ & 001251.11 & -321922.5 & $\mathrm{O}$ & 362.7 & Cho et al. (1996), Haikala (1990) \\
\hline R Cet & 022328.71 & -002411.0 & $\mathrm{O}$ & 166.2 & \\
\hline RT Eri & 033154.33 & -161951.1 & $\mathrm{O}$ & 370.8 & \\
\hline W Eri & 040925.24 & -251543.3 & $\mathrm{O}$ & 376.6 & \\
\hline RS Eri & 041541.82 & -183742.8 & $\mathrm{O}$ & 296.0 & Lewis (1997) \\
\hline R Tau & 042533.46 & +100308.9 & $\mathrm{O}$ & 320.9 & \\
\hline BD Eri & 043111.28 & -000437.6 & $\mathrm{O}$ & 336 & Benson \& Little-Marenin (1996) \\
\hline T Lep & 050243.20 & -215818.7 & $\mathrm{O}$ & 368.1 & \\
\hline U Ori & 055250.95 & +2010 08.1 & $\mathrm{O}$ & 368.3 & see above \\
\hline X Oph & 183557.57 & +084719.9 & $\mathrm{O}$ & 328.9 & see above \\
\hline RT Aql & 193540.42 & +113627.6 & $\mathrm{O}$ & 327.1 & Young (1995) \\
\hline BG Cyg & 193656.87 & +282351.4 & $\mathrm{O}$ & $419.8^{c}$ & \\
\hline Z Cyg & 200002.6 & +495407 & $\mathrm{O}$ & 263.7 & Young (1995), Josselin et al. (1998) \\
\hline RU Cap & 202939.36 & -215139.6 & $\mathrm{O}$ & 347.4 & \\
\hline TU Peg & 214239.21 & +122805.1 & $\mathrm{O}$ & 321.6 & \\
\hline R Peg & 230408.27 & +101622.8 & $\mathrm{O}$ & 378.1 & see above \\
\hline W Peg & 231722.65 & +260018.4 & $\mathrm{O}$ & 345.5 & Young (1995) \\
\hline \multirow[t]{2}{*}{ R Aqr } & 234114.18 & -153341.8 & $\mathrm{O}$ & 387.0 & \\
\hline & & & & SEST $\mathrm{S}$ & ptember 1996 run \\
\hline AFGL 971 & 063416.4 & +032805 & $\mathrm{C}$ & 653 & Loup et al. (1993), Knapp et al. (1998) \\
\hline CL Mon & 065255.9 & +062637 & $\mathrm{C}$ & 497.2 & Loup et al. (1993), Olofsson et al. (1993), Neri et al. (1998) \\
\hline $\mathrm{R} \mathrm{CMi}$ & 070557.56 & +100616.1 & $\mathrm{C}$ & 337.8 & \\
\hline $\mathrm{R}$ Vol & 070632.6 & -725601 & $\mathrm{C}$ & 453.6 & Loup et al. (1993), Olofsson et al. $(1993,1998)$ \\
\hline R Pyx & 084323.8 & -280104 & $\mathrm{C}$ & 364.7 & \\
\hline V Cru & 125336.64 & -573744.1 & $\mathrm{C}$ & 376.5 & \\
\hline R Cap & 200830.18 & -142502.3 & $\mathrm{C}$ & 345.1 & \\
\hline RZ Peg & 220339.05 & +331539.7 & $\mathrm{C}$ & 438.7 & SIMBAD \\
\hline$o$ Cet & 021649.05 & -031213.4 & $\mathrm{O}$ & 332.0 & $\begin{array}{l}\text { Loup et al. (1993), Young (1995), Bujarrabal et al. (1994), } \\
\text { Stanek et al. (1995), Knapp et al. (1998) }\end{array}$ \\
\hline U Ari & 030816.56 & +143641.9 & $\mathrm{O}$ & 371.1 & Young (1995) \\
\hline RT Eri & 033154.33 & -161951.1 & $\mathrm{O}$ & 370.8 & \\
\hline U Hor & 035111.83 & -455840.3 & $\mathrm{O}$ & 348.4 & Haikala et al. (1994) \\
\hline W Eri & 040925.24 & -251543.3 & $\mathrm{O}$ & 376.6 & \\
\hline R Tau & 042533.46 & +100308.9 & $\mathrm{O}$ & 320.9 & \\
\hline RX Tau & 043531.75 & +081413.9 & $\mathrm{O}$ & 331.8 & Duflot et al. (1995) \\
\hline R Cae & 043845.87 & -381951.3 & $\mathrm{O}$ & 391.0 & Haikala et al. (1994) \\
\hline T Lep & 050243.20 & -215818.7 & $\mathrm{O}$ & 368.1 & \\
\hline U Dor & 050950.90 & -642242.6 & $\mathrm{O}$ & 394.4 & Loup et al. (1993) \\
\hline RT Lep & 054028.76 & -234302.7 & $\mathrm{O}$ & 399.0 & \\
\hline U Ori & 055250.95 & +201008.1 & $\mathrm{O}$ & 368.3 & see above \\
\hline AS Pup & 080750.32 & -380134.7 & $\mathrm{O}$ & 324.7 & Haikala et al. (1994) \\
\hline R Car & 093059.21 & -623401.1 & $\mathrm{O}$ & 308.7 & \\
\hline W Vel & 101322.67 & -541344.8 & $\mathrm{O}$ & 394.7 & \\
\hline RZ Mus & 125730.64 & -704135.3 & $\mathrm{O}$ & 333.7 & \\
\hline AQ Cen & 140201.96 & -351531.8 & $\mathrm{O}$ & 387.5 & \\
\hline RU Hya & 140840.87 & -283901.0 & $\mathrm{O}$ & 331.5 & \\
\hline Y Lup & 145556.79 & -544602.7 & $\mathrm{O}$ & 396.8 & \\
\hline S Ser & 151918.97 & +142935.0 & $\mathrm{O}$ & 371.8 & \\
\hline RS Lib & 152124.28 & -224405.4 & $\mathrm{O}$ & 217.7 & Young (1995) \\
\hline BG Ser & 154101.46 & -013311.7 & $\mathrm{O}$ & $143^{d}$ & \\
\hline R Ser & 154823.26 & +151703.1 & $\mathrm{O}$ & 356.4 & see above \\
\hline RR Sco & 165326.31 & -303008.3 & $\mathrm{O}$ & 281.5 & Young (1995) \\
\hline RW Sco & 171134.82 & -332232.5 & $\mathrm{O}$ & 388.5 & \\
\hline V545 Oph & 173643.78 & -231949.4 & $\mathrm{O}$ & 223 & \\
\hline V438 Sco & 175351.45 & -372841.3 & $\mathrm{O}$ & 392.0 & \\
\hline WY Her & 175759.53 & +233541.3 & $\mathrm{O}$ & 376 & \\
\hline X Oph & 183557.57 & $\begin{array}{l}+084719.9 \\
\end{array}$ & $\mathrm{O}$ & 328.9 & see above \\
\hline V342 Sgr & 190921.19 & -325605.4 & $\mathrm{O}$ & 372 & Loup et al. (1993) \\
\hline W Aql & 191241.6 & -070808 & $\mathrm{O}$ & 490.4 & $\begin{array}{l}\text { Loup et al. (1993), Bieging \& Latter (1994), Sahai \& Liechti (1994), } \\
\text { Bieging et al. (1999), Knapp et al. (1998) }\end{array}$ \\
\hline RT Aql & 193540.42 & +113627.6 & $\mathrm{O}$ & 327.1 & see above \\
\hline RR Sgr & 195249.89 & -291923.6 & $\mathrm{O}$ & 336.3 & \\
\hline RR Aql & 195500.41 & $-0201 \quad 17.5$ & $\mathrm{O}$ & 394.8 & Loup et al. (1993), Olofsson et al. (1998) \\
\hline
\end{tabular}


Table 1. continued

\begin{tabular}{|c|c|c|c|c|c|}
\hline Name & $\begin{array}{l}\text { R.A. } \\
(1950)\end{array}$ & $\begin{array}{l}\text { Dec. } \\
(1950)\end{array}$ & Type & $\begin{array}{l}\begin{array}{l}\text { Period } \\
\text { days) }\end{array} \\
\end{array}$ & References \\
\hline \multicolumn{6}{|c|}{ SEST September 1997 run } \\
\hline RZ Peg & 220339.05 & +33 1539.7 & $\mathrm{C}$ & $4 \overline{38.7}$ & \\
\hline$o$ Cet & 021649.05 & -031213.4 & $\mathrm{O}$ & 332.0 & see above \\
\hline RT Eri & 033154.33 & -161951.1 & $\mathrm{O}$ & 370.8 & \\
\hline AQ Cen & 140201.96 & -351531.8 & $\mathrm{O}$ & 387.5 & \\
\hline Y Lup & 145556.79 & -544602.7 & $\mathrm{O}$ & 396.8 & \\
\hline RR Sco & 165326.31 & $\begin{array}{lll}-30 & 30 & 08.3\end{array}$ & $\mathrm{O}$ & 281.5 & see above \\
\hline RW Sco & 171134.82 & -332232.5 & $\mathrm{O}$ & 388.5 & \\
\hline V438 Sco & 175351.45 & -372841.3 & $\mathrm{O}$ & 392.0 & \\
\hline V342 Sgr & 190921.19 & -325605.4 & $\mathrm{O}$ & 372 & see above \\
\hline RR Sgr & 195249.89 & -291923.6 & $\mathrm{O}$ & 336.3 & \\
\hline RR Aql & 195500.41 & -020117.5 & $\mathrm{O}$ & 394.8 & see above \\
\hline \multicolumn{6}{|c|}{ SEST September 1998 run } \\
\hline AFGL 971 & 063416.4 & +032805 & $\mathrm{C}$ & $6 \overline{53 .}$ & see above \\
\hline $\mathrm{R}$ Vol & 070632.6 & -725601 & $\mathrm{C}$ & 453.6 & see above \\
\hline R Cap & 200830.18 & -142502.3 & $\mathrm{C}$ & 345.1 & \\
\hline U Ari & 030816.56 & +143641.9 & $\mathrm{O}$ & 371.1 & see above \\
\hline W Eri & 040925.24 & -251543.3 & $\mathrm{O}$ & 376.6 & \\
\hline R Tau & 042533.46 & +100308.9 & $\mathrm{O}$ & 320.9 & \\
\hline RX Tau & 043531.75 & +08 1413.9 & $\mathrm{O}$ & 331.8 & \\
\hline T Lep & 050243.20 & -215818.7 & $\mathrm{O}$ & 368.1 & \\
\hline U Dor & 050950.90 & -642242.6 & $\mathrm{O}$ & 394.4 & \\
\hline U Ori & 055250.95 & +201008.1 & $\mathrm{O}$ & 368.3 & see above \\
\hline R Car & 093059.21 & -623401.1 & $\mathrm{O}$ & 308.7 & \\
\hline W Vel & 101322.67 & -541344.8 & $\mathrm{O}$ & 394.7 & \\
\hline RZ Mus & 125730.64 & -704135.3 & $\mathrm{O}$ & 333.7 & \\
\hline AQ Cen & 140201.96 & $\begin{array}{lll}-35 & 1531.8\end{array}$ & $\mathrm{O}$ & 387.5 & \\
\hline Y Lup & 145556.79 & -544602.7 & $\mathrm{O}$ & 396.8 & \\
\hline V545 Oph & 173643.78 & -231949.4 & $\mathrm{O}$ & 223 & \\
\hline X Oph & 183557.57 & +084719.9 & $\mathrm{O}$ & 328.9 & see above \\
\hline
\end{tabular}

Notes. (a) Currently (Nov. 1998) listed in SIMBAD as a possibly Semi-Regular (SR) without period. (b) Currently listed in SIMBAD as a SR with a period of 393.4 days. (c) Currently listed in SIMBAD with a period of 291 days! The HIPPARCOS output catalog (ESA 1997) lists a period of 287.3 days. (d) The HIPPARCOS output catalog lists a period of 404 days.

Comparing the SIMBAD database with the periods listed in the 4th edition of the GCVS shows that there are four stars for which the periods changed by more than 4 days: AX Cep (399.1), U Ori (372.5), X Oph (334.2) and Y Lup (402.1).

Some stars have been assigned variable star names (as of Nov. 1998): AFGL 230 (V669 Cas), AFGL 935 (V713 Mon), AFGL 971 (V688 Mon), AFGL 2310 (V1418 Aql), AFGL 2368 (V1420 Aql), CIT 13 (V1426 Cyg).

NRAO 12 m telescope to the expected value for the IRAM$30 \mathrm{~m}$-telescope, assuming a point source, we again found an underestimate of the temperature scale by a factor of 2.2 for the first day of SO observations. Hence, for the first day of SO observations the observed brightness temperatures were multiplied by 2.2 ; for the second day no correction was applied. We estimate the final calibration for all observed lines to be accurate by about $10 \%(1 \sigma)$. This is true for all runs with the IRAM-30 m-telescope.

For the January 1995 run (Observer MG) the ${ }^{12} \mathrm{CO}$ $J=1-0,2-1$ and 3-2 transitions were observed simultaneously with three SIS receivers. We used as backends the $1 \mathrm{MHz}$ filterbank and the autocorrelator, which was split into three parts. The data reduction is similar as described above. For the $J=3-2$ line there were at that time no calibration observations available for the IRAM30 m-telescope.

For the August 1995 run (observer EJ) the ${ }^{12} \mathrm{CO}$ $J=1-0,2-1$ transitions were observed simultaneously, in a set-up identical to the December 1994 run.
The JCMT data were taken on August 28 and September $1 \&$ 7, 1995 (observers FB and RT). The ${ }^{12} \mathrm{CO}$ $J=2-1, J=3-2$ and, for a subsample, ${ }^{13} \mathrm{CO} J=2-1$ and $J=3-2$ were observed using two SIS receivers. The backend was the digital autocorrelation spectrometer. A chopping secondary was used with a throw of $2^{\prime}$. The data reduction is similar as described above. We estimate the calibration to be accurate to about $10 \%(1 \sigma)$.

The first set of SEST data was taken between 11 and 16 September 1996 (observer MG). Either ${ }^{12} \mathrm{CO} J=1-0$ and $\mathrm{SiO}(3-2, v=0)$, or ${ }^{12} \mathrm{CO} J=1-0$ and $\mathrm{CS}(3-2)$ were observed simultaneously using the $2 \mathrm{~mm}$ and $3 \mathrm{~mm}$ SIS receivers. The former setting was used to observe Orich sources, and the latter was used for the C-rich sources. This was not our preferred set-up but the $1.3 \mathrm{~mm}$ receiver was unavailable during our run to observe the CO $(2-1)$ line. The dual beam switching mode was used with a throw of $2^{\prime} 26^{\prime \prime}$ in azimuth. One of two available acousto-optical spectrometers was split and connected to the two receivers with a channel separation of $0.7 \mathrm{MHz}$. 
During the second (20-21 September 1997; observer RS) and third (30 September 1998; observer MG) SEST run the ${ }^{12} \mathrm{CO} J=1-0$ and $2-1$ lines were observed simultaneously with the $1.3 \mathrm{~mm}$ and $3 \mathrm{~mm}$ SIS receivers using the dual beam switching mode as outlined above. We targeted only those stars which had already been detected in the 1996 September run. The data reduction for the SEST runs is similar as described above. Based on a comparison with calibration sources we estimate the brightness temperature scale to be accurate to about $10 \%$ $(1 \sigma)$.

\section{Results of the observations}

Table 3 lists the results of the observing run. Included are the name of the object, the transition, the channel spacing, the rms noise, the peak temperature, the integrated intensity, the central velocity with respect to the local standard of rest and half the velocity width at zero intensity which equals the expansion velocity of the circumstellar shell. These quantities were determined either directly from the profiles or from profile fits using the reduction program CLASS (Forveille et al. 1990). Typical $1 \sigma$ uncertainties in the central velocity and the expansion velocity are $1 \mathrm{~km} \mathrm{~s}^{-1}$. The $1 \sigma$ uncertainties in the peak temperature and the integrated intensity are dominated by the calibration uncertainties. Values flagged with a colon are particularly uncertain. Upper limits are $3 \sigma$ values.

Data of stars observed with the IRAM-30 m-telescope on two separate occasions are co-added first. The results are also included in Table 3. For ZZ Gem we only coadded the $\mathrm{CO}(2-1)$ spectra as the $\mathrm{CO}(1-0)$ line was detected on only one occasion; for R Pyx only the December 1994 data is used, as the integration time during the January 1995 run was only three minutes, resulting in a poor signal-to-noise level. For AX Cep only the January 1995 data is used, as the CO $(2-1)$ emission is much stronger, and the peak of strongest emission was found to be at a slight off-set compared to the position observed in December 1994.

Data obtained with SEST were also co-added, and only the final results are included in Table 3 . In the cases where the CO $(1-0)$ line was detected on two or three occasions, the observed temperatures were in good agreement within the limits of the observation. Also observations of $\mathrm{CO}(1-0)$ and $(2-1)$ calibrator stars on different occasions were in agreement with each other.

For stars without detection the stellar LSR velocity is listed in brackets when available. References are listed in Table 1. When this is "SIMBAD" it is an optical velocity as listed in the "oRV" field, transformed to the LSR frame. When several significantly different values are listed by SIMBAD, the range is given.

The calibrated profiles are shown in the Figs. 1-3 (carbon stars) and 4-9 (oxygen-rich stars). A velocity
Table 2. Lines observed

\begin{tabular}{|c|c|c|c|c|c|}
\hline Line & $\begin{array}{r}\text { Frequency } \\
(\mathrm{MHz}) \\
\end{array}$ & Teles. & $\overline{F_{\text {eff }}}$ & $B_{\text {eff }}$ & $\begin{array}{l}\text { Beam } \\
\left({ }^{\prime \prime}\right)\end{array}$ \\
\hline \multirow[t]{2}{*}{${ }^{12} \mathrm{CO}(1-0)$} & 115271.204 & IRAM & 0.92 & 0.67 & 20.9 \\
\hline & & SEST & & 0.70 & 45 \\
\hline${ }^{13} \mathrm{CO}(2-1)$ & 220396.700 & JCMT & & 0.69 & 22.0 \\
\hline \multirow{3}{*}{${ }^{12} \mathrm{CO}(2-1)$} & 2305 & IRAM & 0.86 & 0.39 & 10.4 \\
\hline & & JCMT & & 0.69 & 21.1 \\
\hline & & SEST & & 0.60 & 23 \\
\hline${ }^{13} \mathrm{CO}(3-2)$ & 330587.959 & JCMT & & 0.58 & 14.7 \\
\hline \multirow{2}{*}{${ }^{12} \mathrm{CO}(3-2)$} & 345795.975 & IRAM & 0.77 & 0.25 & 8.3 \\
\hline & & JCMT & & 0.58 & \\
\hline $\mathrm{HCN}$ & 8863 & IRAM & 0.92 & 0.76 & 27.0 \\
\hline & 13026 & SEST & & 0.69 & 42 \\
\hline CS (3 & 1469 & SEST & & 0.67 & 34 \\
\hline $\mathrm{SO}\left(6_{5}-5_{4}\right)$ & 219949.391 & IRAM & 0.86 & 0.41 & 10.9 \\
\hline
\end{tabular}

range of $100 \mathrm{~km} \mathrm{~s}^{-1}$ is plotted, centered on the system velocity. Stars not detected in any of the observed lines are not shown. Stars observed with different telescopes are shown separately. The figures are arranged by increasing right ascension.

The line shapes of the profiles are that typical of AGB stars, that is, parabolic (e.g. R For in Fig. 1, U Dor in Fig. 5 of AFGL 2968 in Fig. 8), or flat-topped (e.g. AFGL 2310 in Fig. 2). Some line profiles are affected by interstellar emission or absorption (e.g. AFGL 971 in Fig. 2, and AFGL 5625 and V1548 Cyg in Fig. 3). The most unusually shaped line profile is that of AFGL 2368 (Fig. 3), which shows a clear asymmetrical profile, in particular in the $1-0$ line. A SEST CO $(1-0)$ observation (Nyman et al. 1992) does not seem to show this, but there is a hint of a similar asymmetrical line shape in an CO $(1-0)$ observation with the FCRAO (Margulis et al. 1990).

\section{Near-infrared observations}

We obtained JHK photometry for a few stars with the $1.5 \mathrm{~m}$ Carlos Sanchez telescope at the Teide observatory on the island of Tenerife. A single-channel infrared photometer was used with an aperture of $15^{\prime \prime}$. Cycles of "onoff-on" observations were obtained using a throw of $20^{\prime \prime}$. The observation was obtained on November 28, 1998, and the results are listed in Table 4 . For four stars these appear to be the first NIR measurements published, which will be used later on to obtain the distance using a period$M_{K}$-relation.

\section{Analysis}

\subsection{Modelling some SEDs}

A few very red stars without known pulsation period (all observed with the IRAM-30 m-telescope in December 1994) are treated in a different way from the rest of the 
Table 3. The observed quantities

\begin{tabular}{|c|c|c|c|c|c|c|c|c|}
\hline name & transition & $\begin{array}{c}\Delta v \\
\left(\mathrm{~km} \mathrm{~s}^{-1}\right)\end{array}$ & $\begin{array}{l}\mathrm{rms} \\
(\mathrm{K}) \\
\end{array}$ & $\begin{array}{c}T_{\text {peak }} \\
(\mathrm{K}) \\
\end{array}$ & $\begin{array}{c}\int T \mathrm{~d} v \\
\left(\mathrm{~K} \mathrm{~km} \mathrm{~s}^{-1}\right)\end{array}$ & $\begin{array}{c}v_{\mathrm{c}} \\
\left(\mathrm{km} \mathrm{s}^{-1}\right)\end{array}$ & $\begin{array}{c}v_{\mathrm{e}} \\
\left(\mathrm{km} \mathrm{s}^{-1}\right) \\
\end{array}$ & remarks \\
\hline \multicolumn{9}{|c|}{$\underline{\text { IRAM December } 1994 \text { run }}$} \\
\hline & & & C-sta & & & & & \\
\hline \multirow{2}{*}{ V390 Cas } & $1-0$ & 2.60 & 0.018 & - & - & - & - & \\
\hline & $2-1$ & 1.30 & 0.038 & - & - & - & - & \\
\hline \multirow{2}{*}{ PT Cas } & $1-0$ & 2.60 & 0.028 & - & - & - & - & \\
\hline & $2-1$ & 1.30 & 0.050 & - & - & - & - & \\
\hline \multirow{2}{*}{ CN Per } & $1-0$ & 2.60 & 0.020 & - & - & - & - & \\
\hline & $2-1$ & 1.30 & 0.031 & - & - & - & - & \\
\hline \multirow[t]{2}{*}{ R For } & $1-0$ & 1.63 & 0.034 & 0.38 & $11.4 \pm 0.3$ & -1.7 & 16.9 & \\
\hline & $2-1$ & 0.81 & 0.072 & 1.62 & $43.3 \pm 0.5$ & -2.2 & 16.9 & \\
\hline \multirow[t]{4}{*}{ AFGL 5076} & $1-0$ & 1.63 & 0.037 & 0.63 & $16.8 \pm 0.3$ & -66.3 & 20.9 & \\
\hline & $2-1$ & 0.81 & 0.048 & 1.22 & $30.2 \pm 0.3$ & -66.8 & 19.1 & \\
\hline & $\mathrm{HCN}$ & 2.11 & 0.019 & 0.14 & $3.1 \pm 0.2$ & -64.0 & 16.8 & \\
\hline & $\mathrm{SO}$ & 1.36 & 0.040 & - & $<2.5$ & - & - & \\
\hline \multirow{2}{*}{ IRAS $02408+5458$} & $1-0$ & 2.60 & 0.023 & 0.25 & $4.2 \pm 0.18$ & -49.8 & 13.5 & \\
\hline & $2-1$ & 1.30 & 0.049 & 0.56 & $8.3 \pm 0.3$ & -49.7 & 10.4 & \\
\hline \multirow{2}{*}{ GY Per } & $1-0$ & 2.60 & 0.020 & - & - & - & - & \\
\hline & $2-1$ & 1.30 & 0.036 & - & - & - & - & \\
\hline \multirow[t]{4}{*}{ UV Aur } & $1-0$ & 2.60 & 0.012 & - & - & $(-14 /-24)$ & - & \\
\hline & $2-1$ & 1.30 & 0.023 & - & - & - & - & \\
\hline & $\mathrm{HCN}$ & 3.38 & 0.010 & - & - & - & - & \\
\hline & $\mathrm{SO}$ & 1.36 & 0.050 & - & - & - & - & \\
\hline \multirow[t]{4}{*}{ ZZ Gem } & $1-0$ & 2.60 & 0.015 & 0.07 & $0.6 \pm 0.1$ & 63 & - & \\
\hline & $2-1$ & 1.30 & 0.036 & 0.21 & $1.9 \pm 0.13$ & 65.5 & 6.4 & \\
\hline & $\mathrm{HCN}$ & 3.38 & 0.012 & - & $<0.5$ & - & - & \\
\hline & $\mathrm{SO}$ & 1.36 & 0.057 & - & $<0.55$ & - & - & \\
\hline AFGL 935 & $1-0$ & 1.63 & 0.037 & 0.43 & $9.5 \pm 0.3$ & 25.5 & 16.7 & \\
\hline & $2-1$ & 0.81 & 0.057 & 0.84 & $17.9 \pm 0.2$ & 25.4 & 16.5 & \\
\hline IRAS $06582+1507$ & $1-0$ & 1.63 & 0.036 & 0.41 & $8.7 \pm 0.3$ & 21.2 & 15.7 & \\
\hline & $2-1$ & 1.30 & 0.057 & 0.67 & $13.2 \pm 0.4$ & 22.0 & 14.9 & \\
\hline $\mathrm{R} \mathrm{CMi}$ & $1-0$ & 2.60 & 0.037 & - & - & $(19 / 34)$ & - & \\
\hline & $2-1$ & 1.30 & 0.076 & - & - & - & - & \\
\hline VX Gem & $1-0$ & 2.60 & 0.036 & - & - & $(29 / 48)$ & - & \\
\hline & $2-1$ & 1.30 & 0.064 & - & - & - & - & \\
\hline C1698 & $1-0$ & 2.60 & 0.042 & - & - & (79) & - & \\
\hline & $2-1$ & 1.30 & 0.086 & - & - & - & - & \\
\hline R Pyx & $1-0$ & 2.60 & 0.030 & $0.12(:)$ & $2(:)$ & $20(:)$ & - & \\
\hline & $2-1$ & 1.30 & 0.038 & 0.42 & $5.7 \pm 0.2$ & 21.5 & 8.8 & \\
\hline V CrB & $1-0$ & 1.63 & 0.029 & 0.61 & $7.6 \pm 0.16$ & -98.9 & 8.1 & \\
\hline & $2-1$ & 0.81 & 0.040 & 1.87 & $20.0 \pm 0.2$ & -98.7 & 8.1 & \\
\hline V Oph & $1-0$ & 2.60 & 0.023 & $0.08(:)$ & $1.0(:)$ & -28 & - & \\
\hline & $2-1$ & 1.30 & 0.033 & 0.43 & $4.4 \pm 0.14$ & -27.2 & 7.1 & \\
\hline AFGL 2310 & $1-0$ & 1.63 & 0.036 & 0.87 & $41 \pm 1.3$ & 16 & 25 & \\
\hline & $2-1$ & 0.81 & 0.058 & 2.83 & $134 \pm 1.3$ & 15.7 & 27.0 & \\
\hline AFGL 2368 & $1-0$ & 1.63 & 0.030 & 0.56 & $26.5 \pm 0.4$ & 17.1 & 27.1 & \\
\hline & $2-1$ & 0.81 & 0.057 & 1.57 & $70 \pm 1$ & 18.2 & 27.9 & \\
\hline AFGL 2686 & $1-0$ & 1.63 & 0.065 & 0.78 & $32.1 \pm 0.7$ & 0.26 & 25.6 & \\
\hline & $2-1$ & 0.81 & 0.114 & 2.27 & $88.3 \pm 0.8$ & -0.21 & 24.4 & \\
\hline & $\mathrm{HCN}$ & 2.11 & 0.023 & 0.51 & $17.8 \pm 0.2$ & 2.2 & 27.5 & \\
\hline & $\mathrm{SO}$ & 1.36 & 0.056 & - & $<\overline{4.5}$ & - & - & \\
\hline V1549 Cyg & $1-0$ & 1.63 & 0.026 & 0.77 & $16 \pm 0.5$ & $5(:)$ & $17(:)$ & \\
\hline & $2-1$ & 0.81 & 0.043 & 2.41 & $60 \pm 2$ & $5(:)$ & 18.2 & \\
\hline AX Cep & $1-0$ & 2.60 & 0.024 & 0.36 & $7.4 \pm 0.3$ & 13.3 & 13.2 & \\
\hline & $2-1$ & 1.30 & 0.031 & 0.47 & $8.0 \pm 0.6$ & 13.1 & 13.2 & \\
\hline AFGL 5625 & $1-0$ & 1.63 & 0.047 & 1.57 & $39.1 \pm 0.6$ & 2.3 & 18.0 & \\
\hline & $2-1$ & 0.81 & 0.064 & 2.72 & $64.0 \pm 0.6$ & 2.2 & 18.0 & \\
\hline CIT 13 & $1-0$ & 1.63 & 0.052 & 1.24 & $27.7 \pm 0.2$ & -4.8 & 13.1 & \\
\hline & $2-1$ & 0.81 & 0.107 & 2.98 & $58.5 \pm 0.4$ & -4.2 & 14.5 & \\
\hline & $\mathrm{HCN}$ & 2.11 & 0.024 & 0.16 & $3.8 \pm 0.4$ & -2.5 & 20.9 & \\
\hline & $\mathrm{SO}$ & 1.36 & 0.059 & - & $<2.5$ & - & - & \\
\hline $\mathrm{AO}$ Lac & $1-0$ & 2.60 & 0.022 & - & - & - & - & \\
\hline & $2-1$ & 1.30 & 0.038 & - & - & - & - & \\
\hline LS Cas & $1-0$ & 2.60 & 0.023 & - & - & - & - & \\
\hline & $2-1$ & 1.30 & 0.038 & - & - & - & - & \\
\hline & & & O-rich & $\operatorname{tars}$ & & & & \\
\hline AFGL 230 & $1-0$ & 1.63 & 0.021 & $0.14(:)$ & $2.2 \pm 0.7$ & $-55(:)$ & $14(:)$ & \\
\hline & $2-1$ & 0.81 & 0.040 & 0.72 & $12.9 \pm 0.2$ & -54.8 & 13.7 & \\
\hline AFGL 5093 & $1-0$ & 2.60 & 0.026 & 0.08 & $1.3(:)$ & $-38(:)$ & $12(:)$ & \\
\hline & $2-1$ & 1.30 & 0.032 & 0.26 & $3.9 \pm 0.2$ & -37.0 & 11.1 & \\
\hline
\end{tabular}


Table 3. continued

\begin{tabular}{|c|c|c|c|c|c|c|c|c|}
\hline name & transition & $\begin{array}{c}\Delta v \\
\left(\mathrm{~km} \mathrm{~s}^{-1}\right)\end{array}$ & $\begin{array}{c}\mathrm{rms} \\
(\mathrm{K})\end{array}$ & $\begin{array}{c}T_{\text {peak }} \\
(\mathrm{K}) \\
\end{array}$ & $\begin{array}{c}\int T \mathrm{~d} v \\
\left(\mathrm{~K} \mathrm{~km} \mathrm{~s}^{-1}\right)\end{array}$ & $\begin{array}{c}v_{\mathrm{c}} \\
\left(\mathrm{km} \mathrm{s}^{-1}\right)\end{array}$ & $\begin{array}{c}v_{\mathrm{e}} \\
\left(\mathrm{km} \mathrm{s}^{-1}\right)\end{array}$ & remarks \\
\hline \multirow[t]{2}{*}{ AFGL 5097} & $1-0$ & 2.60 & 0.017 & - & $<0.7$ & - & - & \\
\hline & $2-1$ & 2.60 & 0.013 & 0.09 & $1.6 \pm 0.1$ & -54 & 13.9 & \\
\hline \multirow{2}{*}{ NV Aur } & $1-0$ & 1.63 & 0.038 & 1.03 & $30.5 \pm 0.3$ & 3.3 & 18.8 & \\
\hline & $2-1$ & 0.81 & 0.072 & 2.24 & $60.9 \pm 0.4$ & 3.6 & 18.4 & \\
\hline \multirow[t]{2}{*}{ AFGL 712} & $1-0$ & 2.60 & 0.017 & 0.23 & $5.0 \pm 0.13$ & -31.5 & 13.6 & \\
\hline & $2-1$ & 1.30 & 0.026 & 0.25 & $5.5 \pm 0.3$ & -31.3 & 13.2 & \\
\hline \multirow[t]{2}{*}{ IRAS $05284+1945$} & $1-0$ & 2.60 & 0.010 & $0.07(:)$ & $0.8 \pm 0.3$ & $15(:)$ & $15(:)$ & \\
\hline & $2-1$ & 1.30 & 0.035 & $0.12(:)$ & $2.1 \pm 0.2$ & $15(:)$ & $15(:)$ & \\
\hline \multirow{2}{*}{ IRAS 06403-0138 } & $1-0$ & 2.60 & 0.015 & $0.05(:)$ & $0.6(:)$ & - & - & IS contamination \\
\hline & $2-1$ & 1.30 & 0.028 & $0.1(:)$ & $1.4(:)$ & 18 & $10(:)$ & \\
\hline \multirow[t]{2}{*}{ IRAS $07113-2747$} & $1-0$ & 2.60 & 0.029 & 0.09 & $1.8 \pm 0.24$ & 96.1 & 7.6 & \\
\hline & $2-1$ & 1.30 & 0.040 & 0.24 & $2.8 \pm 0.2$ & 96.0 & 8.6 & \\
\hline \multirow{2}{*}{ OH $10.1-0.1$} & $1-0$ & 2.60 & 0.048 & - & - & $(52.3)$ & - & \\
\hline & $2-1$ & 1.30 & 0.052 & - & - & - & - & \\
\hline \multirow[t]{2}{*}{ IRAS $21554+6204$} & $1-0$ & 1.63 & 0.032 & 0.82 & $20.2 \pm 0.4$ & -18.8 & 18.4 & \\
\hline & $2-1$ & 0.81 & 0.044 & 1.29 & $32.0 \pm 0.3$ & -18.8 & 17.6 & \\
\hline \multirow[t]{2}{*}{ AFGL 2885} & $1-0$ & 2.60 & 0.018 & 0.10 & $3(:)$ & -25 & 17 & \\
\hline & $2-1$ & 1.30 & 0.036 & 0.45 & $11.5 \pm 0.6$ & -26.0 & 17.3 & \\
\hline \multirow{2}{*}{ AFGL 2968} & $1-0$ & 2.60 & 0.027 & - & $<2$ & - & - & \\
\hline & $2-1$ & 1.30 & 0.042 & 0.51 & $17.7 \pm 0.4$ & -49.3 & 26.4 & \\
\hline & & IRA & M Janua & y 1995 & & & & \\
\hline & & & C-star & & & & & \\
\hline Y Per & $1-0$ & 2.60 & 0.017 & 0.14 & $1.4 \pm 0.10$ & -6.7 & 7.0 & \\
\hline & $2-1$ & 1.30 & 0.031 & 0.32 & $3.1 \pm 0.14$ & -7.0 & 7.4 & \\
\hline & $3-2$ & 2.17 & 0.059 & $0.16(:)$ & $1.5 \pm 0.1$ & $-5(:)$ & $6.8(:)$ & \\
\hline R Ori & $1-0$ & 2.60 & 0.016 & - & $<0.5$ & - & - & \\
\hline & $2-1$ & 2.60 & 0.043 & $0.12(:)$ & $1.4 \pm 0.7$ & $15(:)$ & $10(:)$ & \\
\hline V Aur & $1-0$ & 2.60 & 0.027 & - & $<2$ & - & - & \\
\hline & $2-1$ & 2.60 & 0.037 & 0.16 & $4.1 \pm 0.5$ & $0(:)$ & $20(:)$ & \\
\hline & $3-2$ & 1.08 & 0.348 & - & $<15$ & - & - & \\
\hline ZZ Gem & $1-0$ & 2.60 & 0.018 & - & $<0.5$ & - & - & \\
\hline & $2-1$ & 1.30 & 0.033 & 0.22 & $2.0 \pm 0.2$ & 64.9 & 7.3 & \\
\hline & $3-2$ & 1.08 & 0.094 & - & $<2$ & - & - & \\
\hline R CMi & $1-0$ & 2.60 & 0.028 & - & - & - & - & \\
\hline & $2-1$ & 1.30 & 0.085 & - & - & - & - & \\
\hline R Pyx & $1-0$ & 2.60 & 0.087 & - & $<2$ & - & - & \\
\hline & $2-1$ & 1.30 & 0.106 & $0.4(:)$ & $4.8 \pm 0.5$ & 21.3(:) & 8.2 & \\
\hline & $3-2$ & 1.08 & 0.388 & - & $<10$ & - & - & \\
\hline V CrB & $1-0$ & 0.81 & 0.033 & 0.57 & $6.92 \pm 0.10$ & -99.3 & 8.2 & \\
\hline & $2-1$ & 1.30 & 0.048 & 2.14 & $23.1 \pm 0.3$ & -98.9 & 8.7 & \\
\hline & $3-2$ & 1.08 & 0.121 & 1.36 & $14.1 \pm 0.48$ & -97.1 & 7.8 & \\
\hline V Oph & $1-0$ & 2.60 & 0.025 & $0.1(:)$ & $<1$ & - & - & \\
\hline & $2-1$ & 1.30 & 0.072 & 0.50 & $5.0 \pm 0.3$ & -27.7 & 6.9 & \\
\hline AX Cep & $1-0$ & 1.63 & 0.027 & 0.37 & $7.83 \pm 0.15$ & 12.9 & 12.1 & \\
\hline & $2-1$ & 1.30 & 0.036 & 0.91 & $16.9 \pm 0.2$ & 13.3 & 12.8 & \\
\hline & $3-2$ & 2.17 & 0.064 & $0.3(:)$ & $4.6 \pm 0.6$ & $15(:)$ & $11(:)$ & \\
\hline & & & O-rich st & & & & & \\
\hline U Ori & $1-0$ & 2.60 & 0.028 & $0.1(:)$ & $1.2(:)$ & $-40(:)$ & $7(:)$ & \\
\hline & $2-1$ & 0.81 & 0.089 & 1.26 & $12.3 \pm 0.8$ & -38.5 & 8.0 & \\
\hline R LMi & $1-0$ & 1.63 & 0.023 & 0.18 & $2.26 \pm 0.10$ & 0.94 & 7.2 & \\
\hline & $2-1$ & 1.30 & 0.034 & 1.25 & $14.9 \pm 0.17$ & 0.04 & 8.6 & \\
\hline & $3-2$ & 1.08 & 0.099 & 1.03 & $10.9 \pm 0.5$ & 2.2 & 8.2 & \\
\hline R Leo & $1-0$ & 1.63 & 0.026 & 0.34 & $3.87 \pm 0.13$ & -0.58 & 7.2 & \\
\hline & $2-1$ & 1.30 & 0.033 & $2.6(:)$ & $28.6 \pm 0.3$ & -0.63 & 7.2 & \\
\hline & $3-2$ & 1.08 & 0.075 & 2.07 & $19.8 \pm 0.4$ & 1.1 & 7.5 & \\
\hline RS Vir & $1-0$ & 0.81 & 0.038 & 0.24 & $2.31 \pm 0.10$ & -14.0 & 5.7 & \\
\hline & $2-1$ & 0.81 & 0.092 & 2.25 & $19.5 \pm 0.3$ & -13.5 & 6.7 & \\
\hline R Ser & $1-0$ & 1.63 & 0.024 & 0.16 & $1.37 \pm 0.10$ & 31.6 & $5.3(:)$ & \\
\hline & $2-1$ & 0.81 & 0.059 & 1.84 & $13.2 \pm 0.3$ & 31.3 & 6.4 & \\
\hline X Oph & $1-0$ & 1.63 & 0.038 & - & $<0.8$ & - & - & \\
\hline & $2-1$ & 0.81 & 0.079 & 0.93 & $7.3 \pm 0.3$ & -54.9 & 6.3 & \\
\hline R Peg & $1-0$ & 1.63 & 0.027 & $0.08(:)$ & $0.7 \pm 0.1$ & $25.5(:)$ & $7.3(:)$ & \\
\hline & $2-1$ & 1.30 & 0.025 & 0.68 & $5.77 \pm 0.10$ & 24.4 & 6.0 & \\
\hline & $3-2$ & 1.08 & 0.065 & 0.64 & $5.2 \pm 0.2$ & 25.6 & 6.6 & \\
\hline & & $\underline{\mathrm{IRA}}$ & August & 1995 run & & & & \\
\hline & & & D-rich st & & & & & \\
\hline R Cet & $1-0$ & 2.60 & 0.025 & 0.15 & $1.8 \pm 0.1$ & 32.2 & 9.9 & \\
\hline & $2-1$ & 1.30 & 0.035 & 0.37 & $3.9 \pm 0.1$ & 31.9 & 8.6 & \\
\hline
\end{tabular}


Table 3. continued

\begin{tabular}{|c|c|c|c|c|c|c|c|c|}
\hline name & transition & $\begin{array}{c}\Delta v \\
\left(\mathrm{~km} \mathrm{~s}^{-1}\right)\end{array}$ & $\begin{array}{l}\mathrm{rms} \\
(\mathrm{K})\end{array}$ & $\begin{array}{c}T_{\text {peak }} \\
(\mathrm{K}) \\
\end{array}$ & $\begin{array}{c}\int T \mathrm{~d} v \\
\left(\mathrm{~K} \mathrm{~km} \mathrm{~s}^{-1}\right)\end{array}$ & $\begin{array}{c}v_{\mathrm{c}} \\
\left(\mathrm{km} \mathrm{s}^{-1}\right)\end{array}$ & $\begin{array}{c}v_{\mathrm{e}} \\
\left(\mathrm{km} \mathrm{s}^{-1}\right)\end{array}$ & remarks \\
\hline \multirow[t]{2}{*}{ R Tri } & $1-0$ & 2.60 & 0.016 & - & - & $(56 / 64)$ & - & \\
\hline & $2-1$ & 1.30 & 0.026 & - & - & - & - & \\
\hline \multirow[t]{2}{*}{ R Tau } & $1-0$ & 2.60 & 0.023 & - & - & - & - & \\
\hline & $2-1$ & 1.30 & 0.043 & 0.26 & $1.8 \pm 0.1$ & 14.1 & 5.6 & \\
\hline \multirow[t]{2}{*}{ T Lep } & $1-0$ & 2.60 & 0.037 & - & - & - & - & \\
\hline & $2-1$ & 1.30 & 0.055 & $0.1(:)$ & $0.6(:)$ & $-27(:)$ & $6.8(:)$ & \\
\hline \multirow[t]{2}{*}{ X Нyа } & $1-0$ & 2.60 & 0.049 & - & - & - & - & \\
\hline & $2-1$ & 1.30 & 0.053 & 0.23 & $1.9 \pm 0.2$ & 27.0 & 6.8 & \\
\hline \multirow[t]{2}{*}{ R Aqr } & $1-0$ & 2.60 & 0.027 & - & - & - & - & \\
\hline & $2-1$ & 1.30 & 0.033 & 0.073 & $1.5 \pm 0.2$ & -30.4 & 16.7 & \\
\hline \multicolumn{9}{|c|}{ IRAM combined December 1994 \& January 1995 run } \\
\hline ZZ Gem & $2-\overline{1}$ & 1.30 & 0.026 & 0.24 & $2.1 \pm 0.1$ & 65.0 & 6.9 & \\
\hline \multirow[t]{2}{*}{$\mathrm{R}$ CMi } & $1-0$ & 2.60 & 0.022 & - & - & - & - & \\
\hline & $2-1$ & 1.30 & 0.057 & - & - & - & - & \\
\hline \multirow[t]{2}{*}{$\mathrm{V} \mathrm{CrB}$} & $1-0$ & 1.63 & 0.019 & 0.59 & $7.2 \pm 0.1$ & -98.9 & 8.2 & \\
\hline & $2-1$ & 1.30 & 0.025 & 1.91 & $21.9 \pm 0.2$ & -98.9 & 8.3 & \\
\hline \multirow[t]{2}{*}{ V Oph } & $1-0$ & 2.60 & 0.018 & 0.1 & $0.7(:)$ & -27.6 & $8(:)$ & \\
\hline & $2-1$ & 1.30 & 0.032 & 0.44 & $4.7 \pm 0.1$ & -27.3 & 7.8 & \\
\hline \multicolumn{8}{|c|}{ JCMT August 1995 run } & \\
\hline & & & & stars & & & & \\
\hline \multirow[t]{3}{*}{ R For } & $12 / 2-1$ & 0.81 & 0.036 & 0.8 & 20.6 & -2.1 & 17.8 & \\
\hline & $13 / 2-1$ & 1.70 & 0.019 & - & $1.1(:)$ & - & - & \\
\hline & $12 / 3-2$ & 1.08 & 0.082 & 1.2 & 28.5 & -2.0 & 19.0 & \\
\hline UV Aur & $2-1$ & 0.81 & 0.048 & - & - & - & - & \\
\hline \multirow[t]{2}{*}{ R Cap } & $2-1$ & 0.81 & 0.046 & 0.25 & 2.52 & 2.6 & 10.5 & \\
\hline & $3-2$ & 1.08 & 0.083 & 0.35 & 2.4 & 5.6 & $8(:)$ & \\
\hline \multirow[t]{3}{*}{ AX Cep } & $2-1$ & 0.81 & 0.060 & 0.40 & 6.5 & 13.1 & 13.0 & \\
\hline & $3-2$ & 1.08 & 0.109 & 0.35 & 2.2 & 15.1 & - & \\
\hline & \multicolumn{7}{|c|}{ O-rich stars } & \\
\hline $\mathrm{S} \mathrm{Scl}$ & $2-1$ & 0.81 & 0.064 & - & - & $(8.5)$ & - & \\
\hline R Cet & $2-1$ & 0.81 & 0.053 & 0.2 & 2.1 & 32.1 & 7.5 & \\
\hline RT Eri & $2-1$ & 0.81 & 0.054 & 0.15 & 1.4 & 24.7 & 8.5 & \\
\hline W Eri & $2-1$ & 0.81 & 0.040 & 0.18 & 1.9 & 0.2 & 10.0 & \\
\hline RS Eri & $2-1$ & 0.81 & 0.055 & - & - & $(49)$ & - & \\
\hline R Tau & $2-1$ & 0.81 & 0.050 & 0.2 & 1.4 & 14.6 & 5.0 & \\
\hline BD Eri & $2-1$ & 0.81 & 0.051 & - & - & $(-11)$ & - & \\
\hline T Lep & $2-1$ & 0.81 & 0.058 & - & - & - & - & \\
\hline \multirow[t]{4}{*}{ U Ori } & $12 / 2-1$ & 0.81 & 0.033 & 0.6 & 5.2 & -38.6 & 9.7 & \\
\hline & $13 / 2-1$ & 0.85 & 0.019 & $0.06(:)$ & $0.49(:)$ & -40.5 & - & \\
\hline & $12 / 3-2$ & 1.08 & 0.102 & 1.5 & 13.5 & -37.8 & 9.2 & \\
\hline & $13 / 3-2$ & 1.13 & 0.057 & $0.06(:)$ & $0.7(:)$ & - & - & \\
\hline \multirow[t]{2}{*}{ X Oph } & $2-1$ & 0.81 & 0.051 & 0.35 & 2.12 & -55.0 & 6.3 & \\
\hline & $3-2$ & 1.08 & 0.091 & 0.60 & 3.77 & -54.9 & 6.5 & \\
\hline RT Aql & $2-1$ & 0.81 & 0.026 & 0.32 & 2.1 & -29.5 & 6.5 & \\
\hline BG Cyg & $2-1$ & 0.81 & 0.021 & 0.15 & 1.4 & -97.6 & 5.0 & \\
\hline Z Cyg & $2-1$ & 0.81 & 0.054 & 0.26 & 1.8 & -148.4 & 4.5 & \\
\hline RU Cap & $2-1$ & 0.81 & 0.031 & $0.1(:)$ & $0.7(:)$ & $7.5(:)$ & $7.5(:)$ & \\
\hline TU Peg & $2-1$ & 0.81 & 0.030 & $0.1(:)$ & 0.9 & 12.4 & $8.0(:)$ & \\
\hline \multirow[t]{2}{*}{ R Peg } & $2-1$ & 0.81 & 0.036 & 0.24 & 2.0 & 24.3 & 6.5 & \\
\hline & $3-2$ & 1.08 & 0.175 & $0.4(:)$ & $4.0(:)$ & 16.5 & - & \\
\hline \multirow[t]{2}{*}{ W Peg } & $2-1$ & 0.81 & 0.027 & 0.3 & 3.6 & -14.8 & 8.1 & \\
\hline & $3-2$ & 1.08 & 0.109 & 0.6 & 4.8 & -16.4 & 8.1 & \\
\hline R Aqr & $2-1$ & 0.81 & 0.054 & - & - & - & - & \\
\hline
\end{tabular}


Table 3. continued

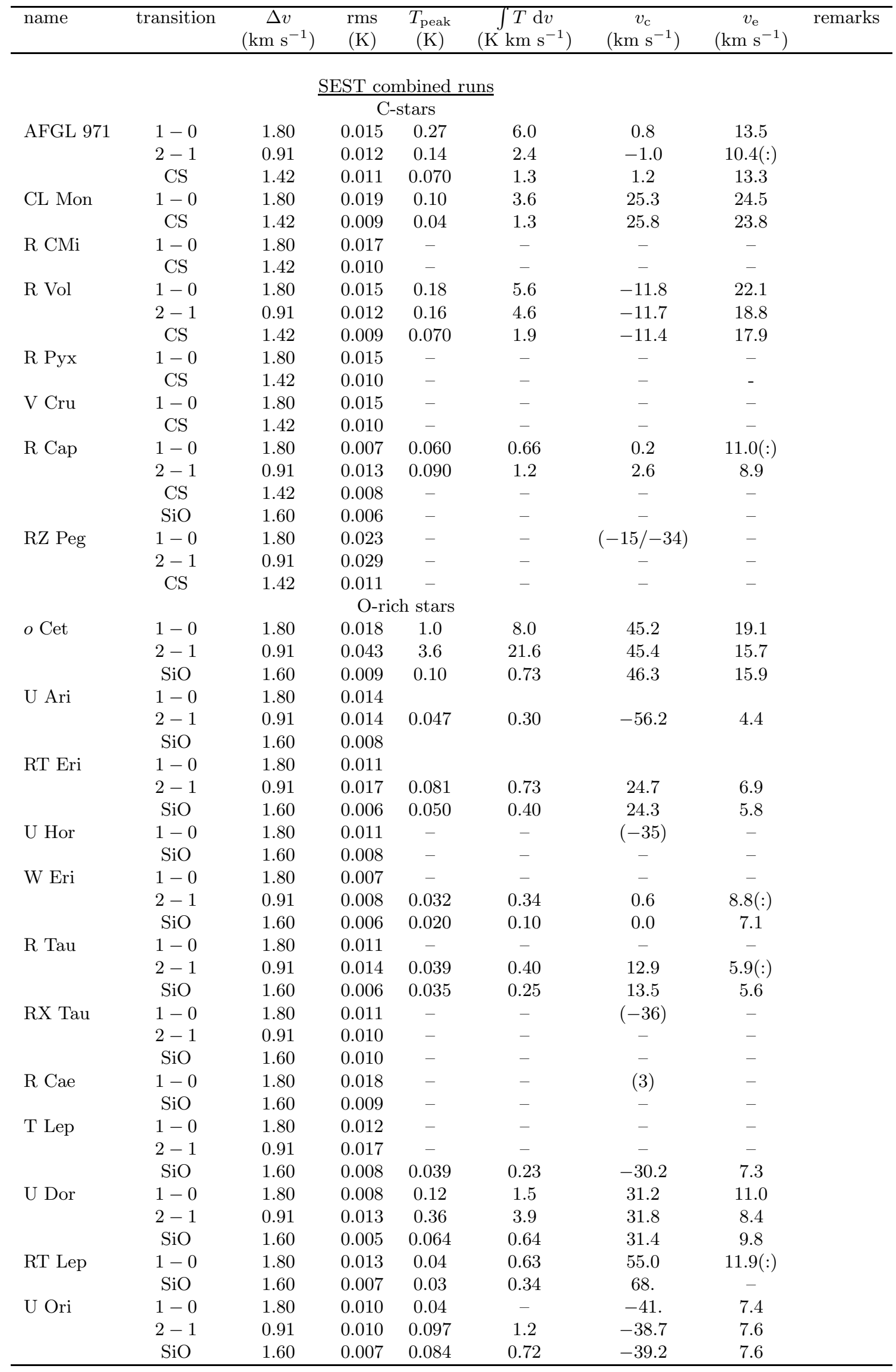


Table 3. continued

\begin{tabular}{|c|c|c|c|c|c|c|c|c|}
\hline name & transition & $\begin{array}{c}\Delta v \\
\left(\mathrm{~km} \mathrm{~s}^{-1}\right)\end{array}$ & $\begin{array}{l}\mathrm{rms} \\
(\mathrm{K})\end{array}$ & $\begin{array}{c}T_{\text {peak }} \\
(\mathrm{K})\end{array}$ & $\begin{array}{c}\int T \mathrm{~d} v \\
\left(\mathrm{~K} \mathrm{~km} \mathrm{~s}^{-1}\right)\end{array}$ & $\begin{array}{c}v_{\mathrm{c}} \\
\left(\mathrm{km} \mathrm{s}^{-1}\right)\end{array}$ & $\begin{array}{c}v_{\mathrm{e}} \\
\left(\mathrm{km} \mathrm{s}^{-1}\right)\end{array}$ & remarks \\
\hline \multirow{2}{*}{ AS Pup } & $1-0$ & 1.80 & 0.015 & - & - & $(-33)$ & - & \\
\hline & $\mathrm{SiO}$ & 1.60 & 0.008 & - & - & - & - & \\
\hline \multirow[t]{3}{*}{ R Car } & $1-0$ & 1.80 & 0.013 & - & - & - & - & \\
\hline & $2-1$ & 0.91 & 0.017 & - & - & - & - & \\
\hline & $\mathrm{SiO}$ & 1.60 & 0.008 & 0.047 & 0.40 & 5.2 & $6.8(:)$ & \\
\hline \multirow[t]{3}{*}{ W Vel } & $1-0$ & 1.80 & 0.010 & - & - & - & - & IS contamination \\
\hline & $2-1$ & 0.91 & 0.016 & - & - & - & - & IS contamination \\
\hline & $\mathrm{SiO}$ & 1.60 & 0.005 & 0.029 & 0.24 & -6.5 & 6.4 & \\
\hline \multirow{3}{*}{ RZ Mus } & $1-0$ & 1.80 & 0.009 & 0.080 & 1.64 & -47.5 & 18.9 & \\
\hline & $2-1$ & 0.91 & 0.014 & 0.054 & 1.14 & -45.5 & 15.5 & \\
\hline & $\mathrm{SiO}$ & 1.60 & 0.006 & - & - & - & - & \\
\hline \multirow[t]{3}{*}{ AQ Cen } & $1-0$ & 1.80 & 0.016 & - & - & - & - & \\
\hline & $2-1$ & 0.91 & 0.036 & 0.11 & 1.0 & 9.1 & 10.2 & \\
\hline & $\mathrm{SiO}$ & 1.60 & 0.008 & 0.043 & 0.49 & 5.0 & 9.5 & \\
\hline \multirow[t]{2}{*}{ RU Hya } & $1-0$ & 1.80 & 0.015 & 0.048 & 0.65 & -24.2 & $7.4(:)$ & \\
\hline & $\mathrm{SiO}$ & 1.60 & 0.008 & - & - & - & - & \\
\hline \multirow[t]{3}{*}{ Y Lup } & $1-0$ & 1.80 & 0.014 & - & - & - & - & \\
\hline & $2-1$ & 0.91 & 0.021 & 0.053 & 0.97 & 17.1 & 13.8 & \\
\hline & $\mathrm{SiO}$ & 1.60 & 0.008 & 0.040 & 0.32 & 17.6 & 11.3 & \\
\hline \multirow[t]{2}{*}{ S Ser } & $1-0$ & 1.80 & 0.017 & - & - & - & - & \\
\hline & $\mathrm{SiO}$ & 1.60 & 0.008 & 0.021 & 0.20 & 23.4 & $8.8(:)$ & \\
\hline \multirow[t]{2}{*}{ RS Lib } & $1-0$ & 1.80 & 0.015 & - & - & - & - & \\
\hline & $\mathrm{SiO}$ & 1.60 & 0.008 & 0.060 & 0.31 & 7.0 & 4.5 & \\
\hline \multirow[t]{2}{*}{ BG Ser } & $1-0$ & 1.80 & 0.015 & - & - & - & - & \\
\hline & $\mathrm{SiO}$ & 1.60 & 0.009 & 0.029 & 0.53 & 5.5 & $11(:)$ & \\
\hline \multirow[t]{2}{*}{ R Ser } & $1-0$ & 1.80 & 0.015 & 0.073 & 0.57 & 29.7 & 6.6 & \\
\hline & $\mathrm{SiO}$ & 1.60 & 0.007 & 0.041 & 0.35 & 31.8 & 6.1 & \\
\hline \multirow[t]{3}{*}{ RR Sco } & $1-0$ & 1.80 & 0.012 & - & - & - & - & \\
\hline & $2-1$ & 0.91 & 0.018 & - & - & - & - & \\
\hline & $\mathrm{SiO}$ & 1.60 & 0.008 & 0.031 & 0.12 & -31.3 & 5.3 & \\
\hline \multirow[t]{3}{*}{ RW Sco } & $1-0$ & 1.80 & 0.011 & 0.043 & 0.48 & -70.3 & 11.0 & \\
\hline & $2-1$ & 0.91 & 0.016 & 0.094 & 1.30 & -70.7 & 10.5 & \\
\hline & $\mathrm{SiO}$ & 1.60 & 0.004 & 0.037 & 0.44 & -70.7 & 10.6 & \\
\hline \multirow[t]{3}{*}{ V545 Oph } & $1-0$ & 1.80 & 0.011 & - & - & - & - & \\
\hline & $2-1$ & 0.91 & 0.011 & 0.060 & 0.63 & -18.7 & 9.3 & \\
\hline & $\mathrm{SiO}$ & 1.60 & 0.009 & - & - & - & - & \\
\hline \multirow[t]{3}{*}{ V438 Sco } & $1-0$ & 1.80 & 0.015 & - & - & - & - & \\
\hline & $2-1$ & 0.91 & 0.018 & - & - & - & - & \\
\hline & $\mathrm{SiO}$ & 1.60 & 0.011 & - & - & - & - & \\
\hline \multirow[t]{2}{*}{ WY Her } & $1-0$ & 1.80 & 0.013 & 0.045 & 0.62 & 0.3 & 11.7 & \\
\hline & $\mathrm{SiO}$ & 1.60 & 0.006 & 0.022 & 0.15 & 1.8 & 9.0 & \\
\hline X Oph & $1-0$ & 1.80 & 0.012 & - & - & - & - & \\
\hline & $2-1$ & 1.82 & 0.015 & - & - & - & - & \\
\hline & $\mathrm{SiO}$ & 1.60 & 0.008 & 0.020 & 0.1 & -57.6 & $5.5(:)$ & \\
\hline V342 Sgr & $1-0$ & 1.80 & 0.018 & 0.20 & 4.9 & 39.2 & 16.1 & \\
\hline & $2-1$ & 0.91 & 0.036 & 0.51 & 11.3 & 38.4 & 15.2 & \\
\hline & $\mathrm{SiO}$ & 1.60 & 0.007 & 0.095 & 1.7 & 39.9 & 15.2 & \\
\hline W Aql & $1-0$ & 1.80 & 0.036 & 1.0 & 30.5 & -25.4 & 20.7 & \\
\hline & $\mathrm{SiO}$ & 1.60 & 0.014 & 0.18 & 4.9 & -26.5 & 17.9 & \\
\hline RT Aql & $1-0$ & 1.80 & 0.010 & 0.047 & 0.53 & -30.8 & $9.5(:)$ & \\
\hline & $\mathrm{SiO}$ & 1.60 & 0.006 & 0.047 & 0.26 & -30.2 & 6.5 & \\
\hline RR Sgr & $1-0$ & 1.80 & 0.012 & & - & - & - & \\
\hline & $2-1$ & 0.91 & 0.018 & 0.18 & 1.2 & 95.3 & 5.2 & \\
\hline & $\mathrm{SiO}$ & 1.60 & 0.006 & 0.029 & 0.24 & 96.0 & 5.6 & \\
\hline RR Aql & $1-0$ & 1.80 & 0.020 & 0.21 & 2.9 & 27.1 & 10.0 & \\
\hline & $2-1$ & 0.91 & 0.031 & 0.69 & 8.2 & 27.9 & 8.7 & \\
\hline & $\mathrm{SiO}$ & 1.60 & 0.008 & 0.13 & 1.33 & 27.3 & 10.2 & \\
\hline
\end{tabular}



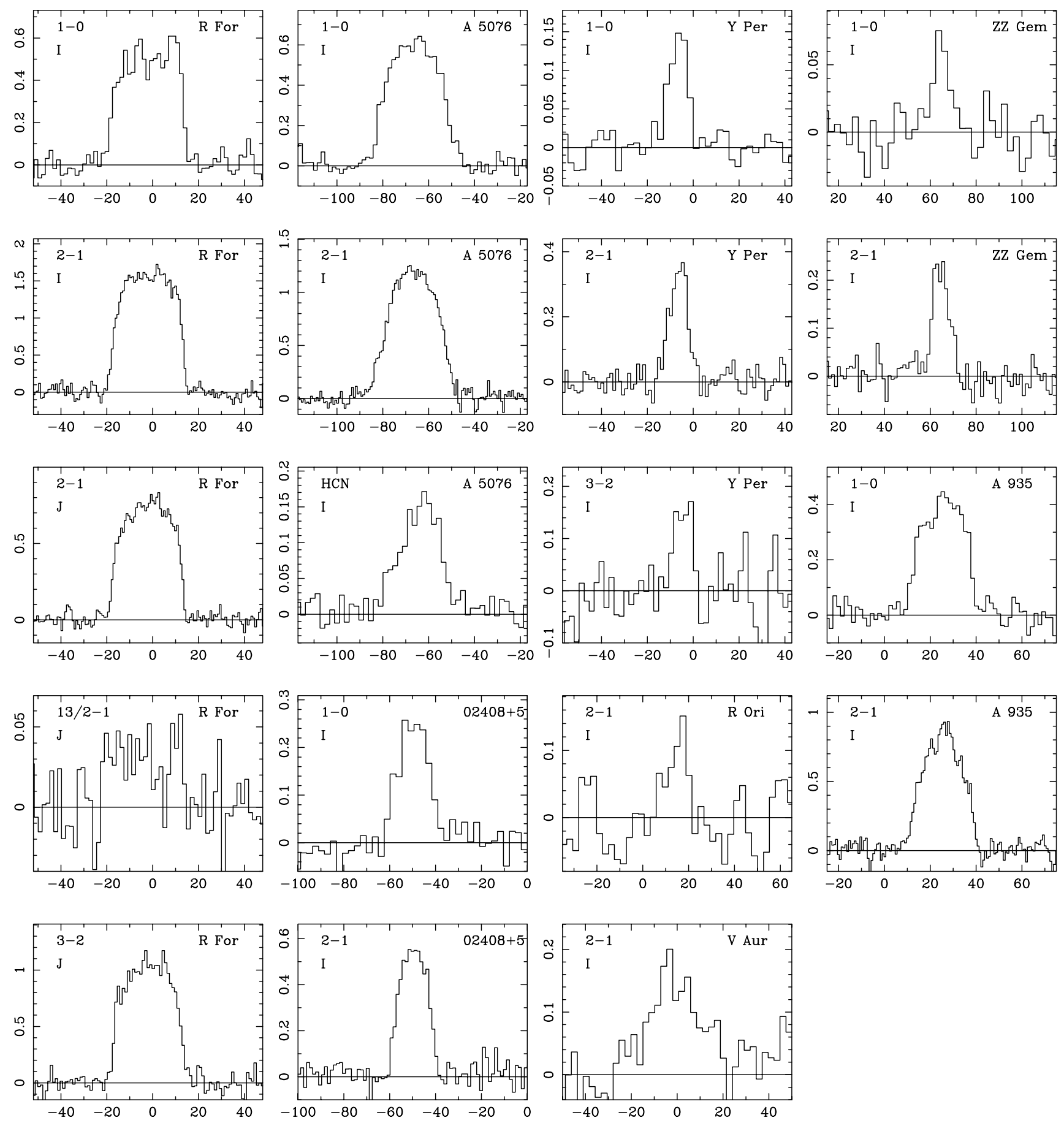

Fig. 1. Line profiles of the carbon stars. Velocity scale is relative to the LSR. Temperatures are on main-beam scale. In the upper left-hand corner the transition, and telescope is indicated: "I", "J", "S", stand for IRAM, JCMT and SEST, respectively

sample. The reason is that for some objects no nearIR data and/or pulsation period is available and the methods discussed below could not be applied.

The object $\mathrm{OH} 10.1-0.1$ (IRAS $18052-2016$ ) is located near the galactic centre and thus we assume a distance of $8.0 \mathrm{kpc}$. The other objects (IRAS $02408+5458$, $06582+1507,05284+1945,06403-0138,21554+6204$, $22480+6002=$ AFGL 2968) are all located outside the solar circle, and were studied by Blommaert et al. (1993). Following Blommaert et al. (1993), kinematic distances could be derived for all objects except IRAS 05284+1945, which in the following we assume to be located at $5 \mathrm{kpc}$, for otherwise its luminosity would be too low to be on the AGB. Jiang et al. (1997) put this object at $9.8 \mathrm{kpc}$ (for their assumed typical AGB luminosity of $8000 L_{\odot}$ ). 

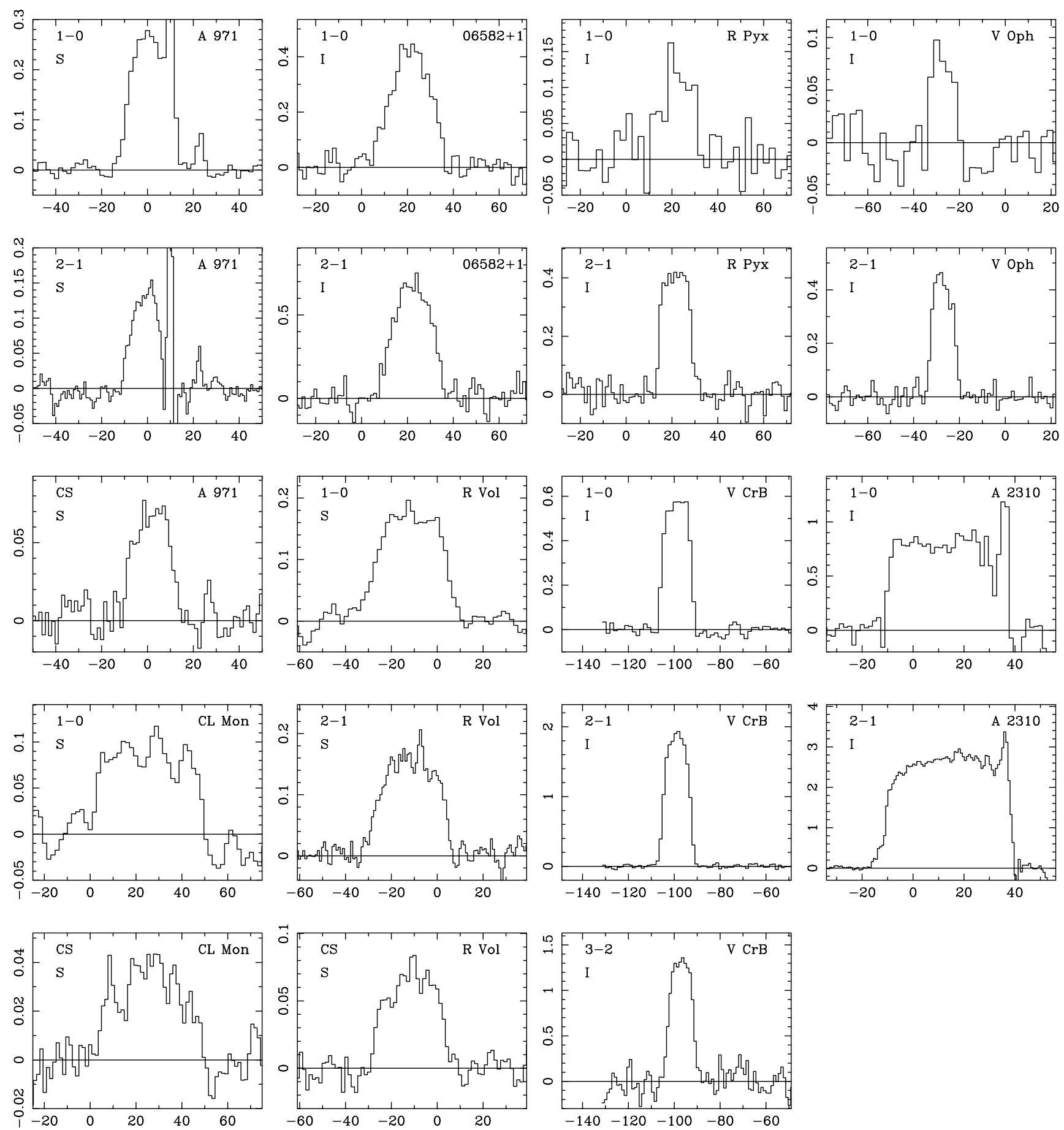

Fig. 2. As Fig. 1

As a next step the SEDs and LRS spectra were fitted using the dust radiative transfer model of Groenewegen (1993; also see Groenewegen et al. 1998). IRAS LRS spectra could be retrieved from the database maintained at the University of Calgary (see http://www.iras.ucalgary.ca/ volk/getlrs_plot.html), except for IRAS $05284+1945$. Broadband data were taken from the IRAS Point Source Catalog $(12,25,60$,
$100 \mu \mathrm{m}$ data), and IR data from Blommaert et al. (1993) (typically JHKLM and narrow-band data near $10 \mu \mathrm{m}$ in the best cases, but note that no near-IR data is available for IRAS $18052-2016$ and $21554+6204$ ). For IRAS $05284+1945$ a $K$ observation was taken from from Garcia-Lario et al. (1997) and $H, K$ observations from Jiang et al. (1997). For AFGL 2968, I, $K$ observations were added from the IRC survey. 

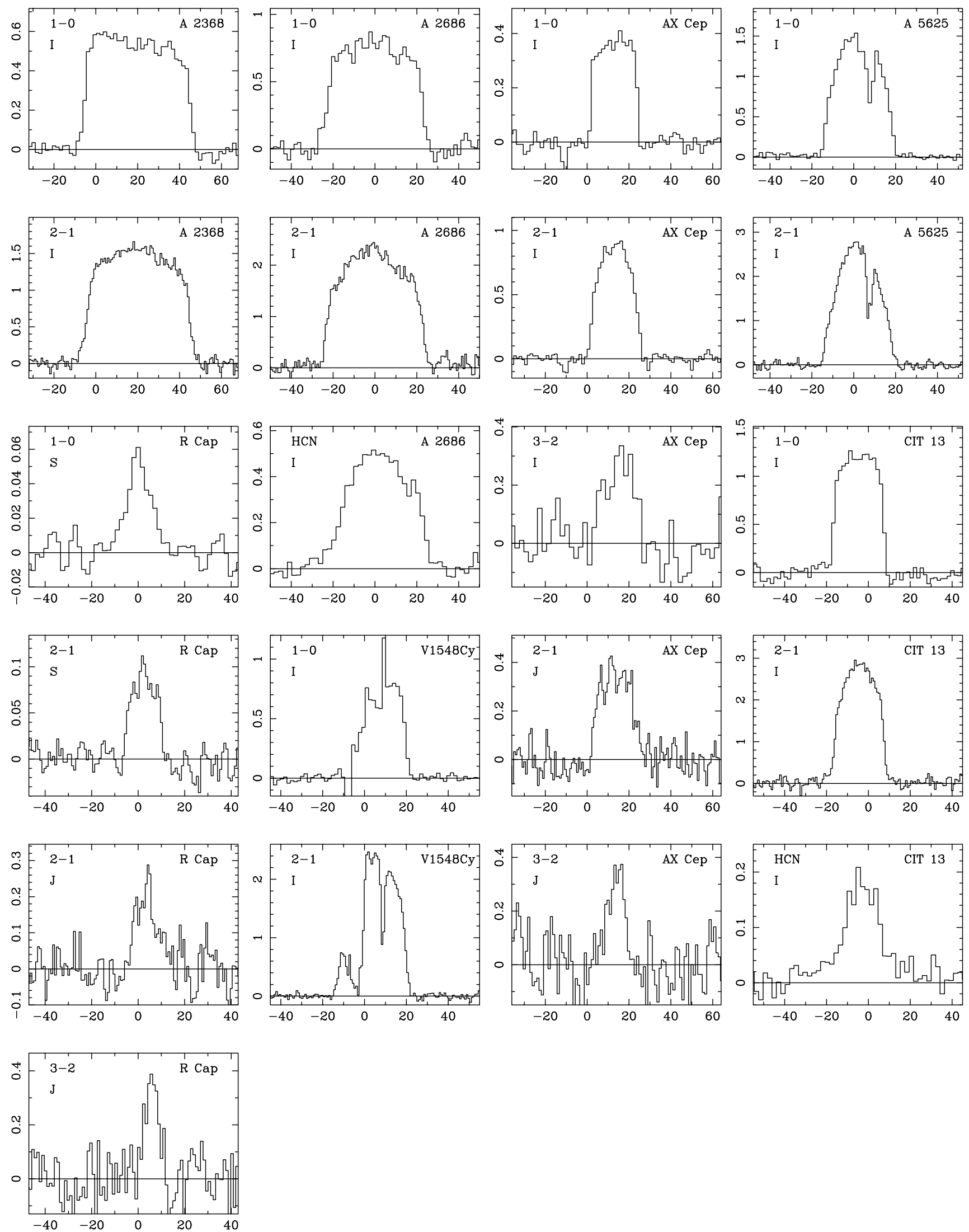

Fig. 3. As Fig. 1 

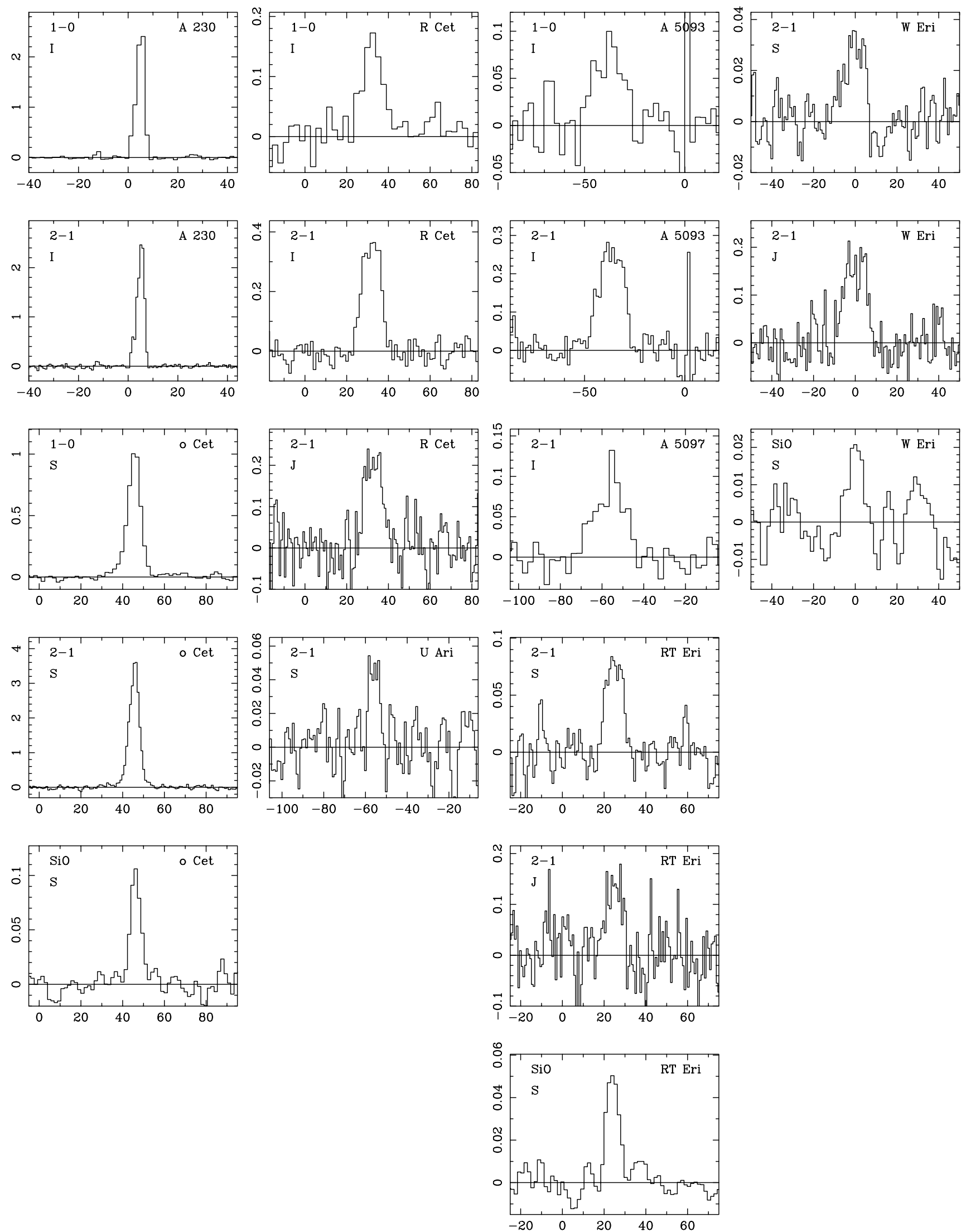

Fig. 4. As Fig. 1, but for the oxygen-rich stars 

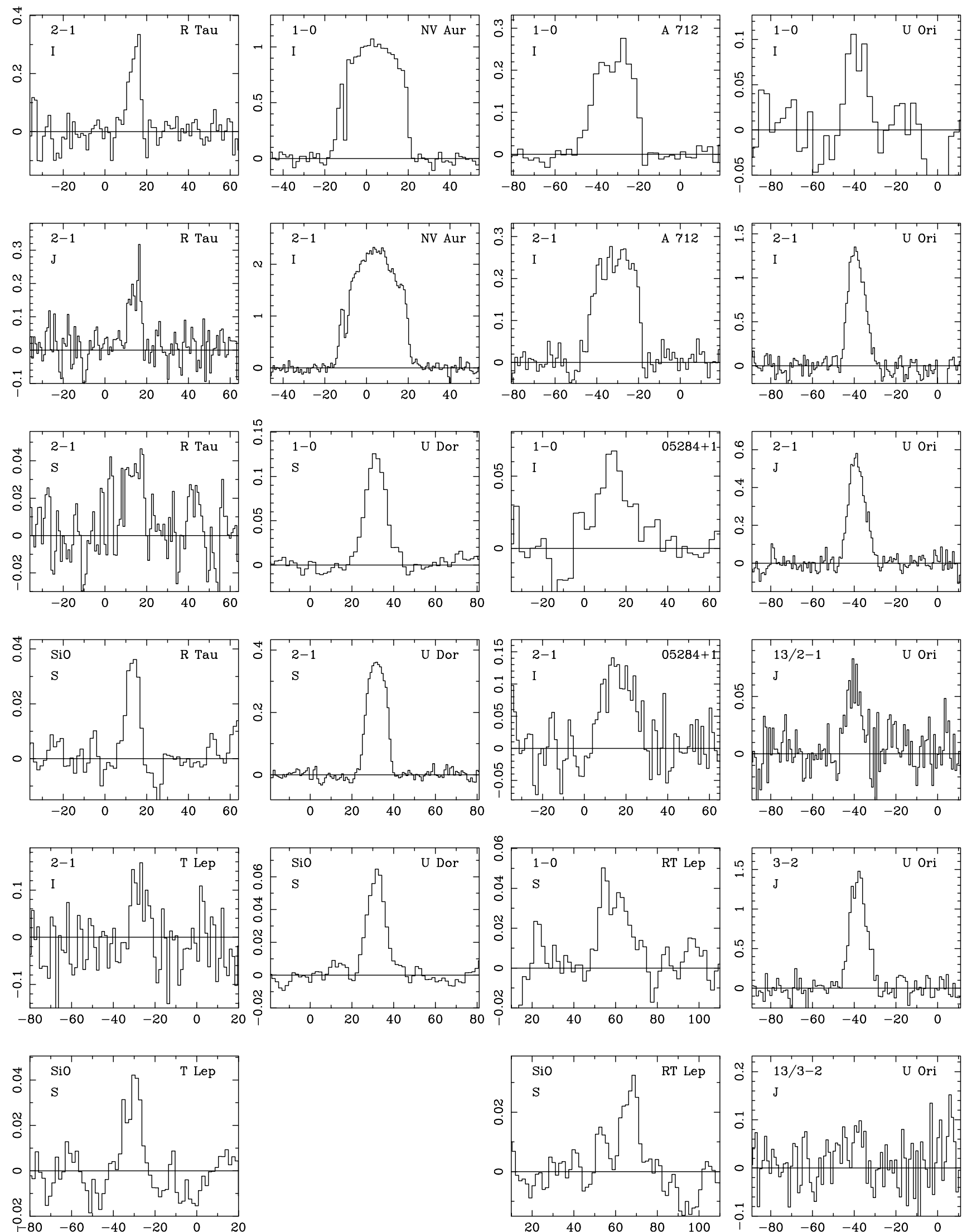

Fig. 5. As Fig. 4 

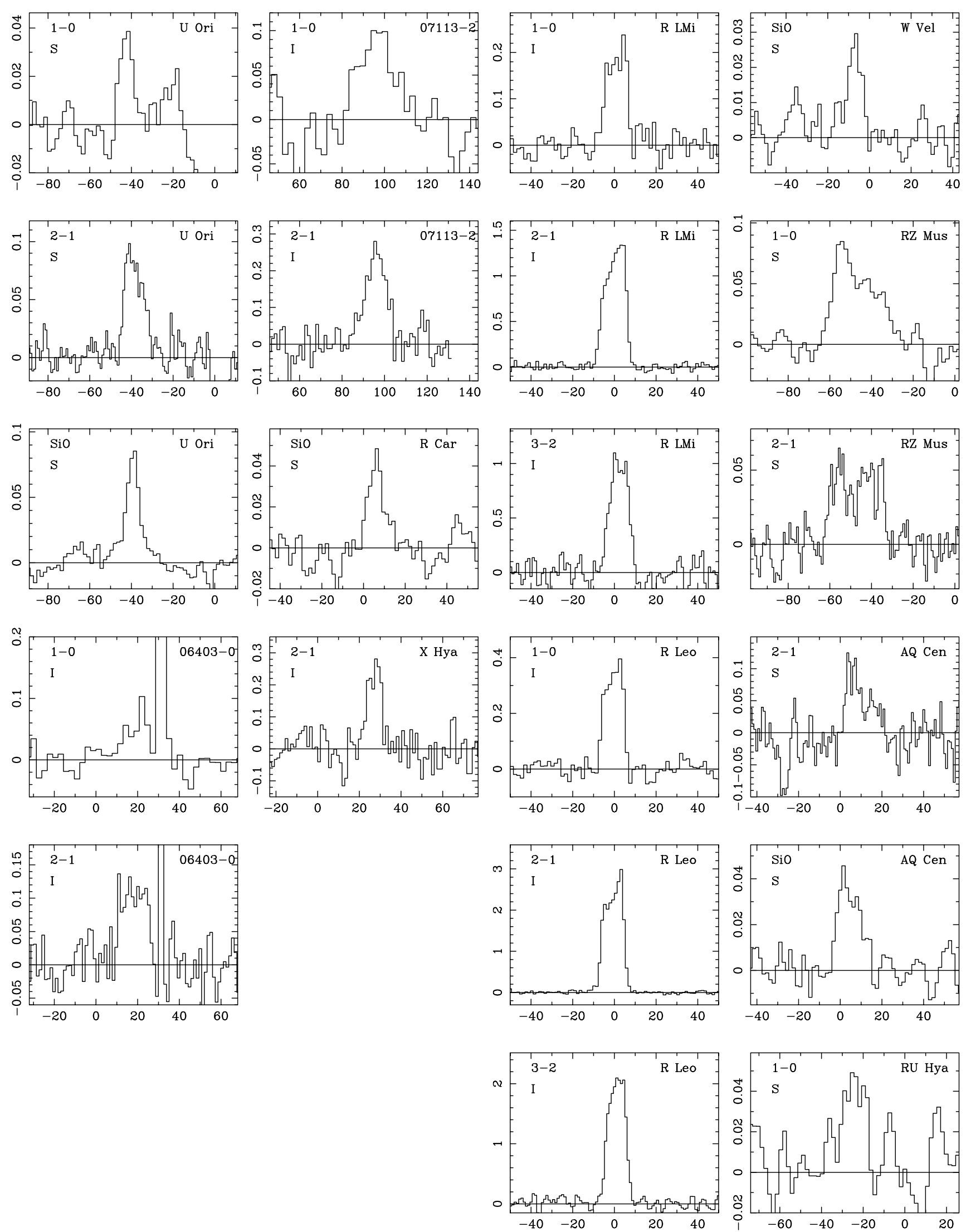

Fig. 6. As Fig. 4 

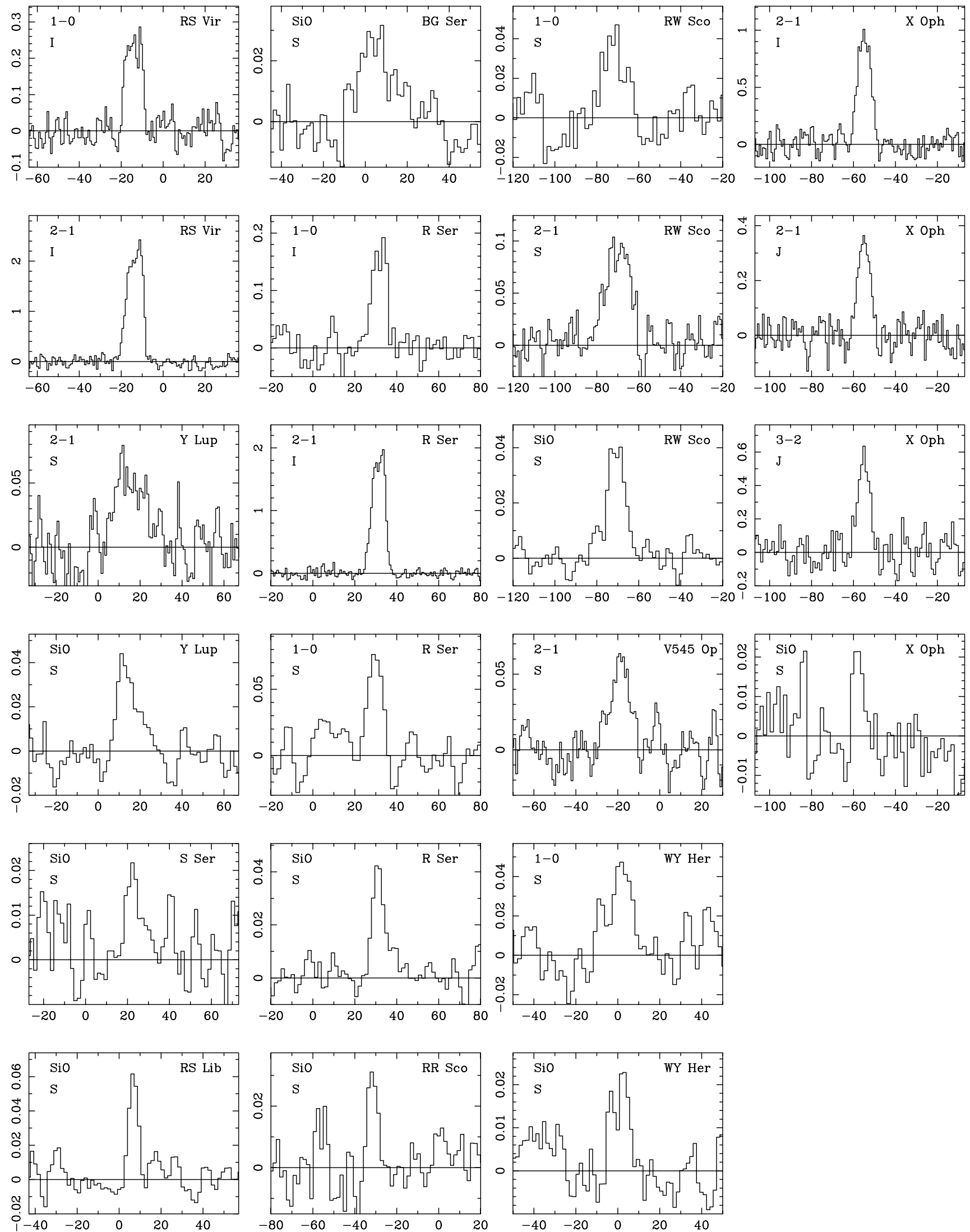

Fig. 7. As Fig. 4 

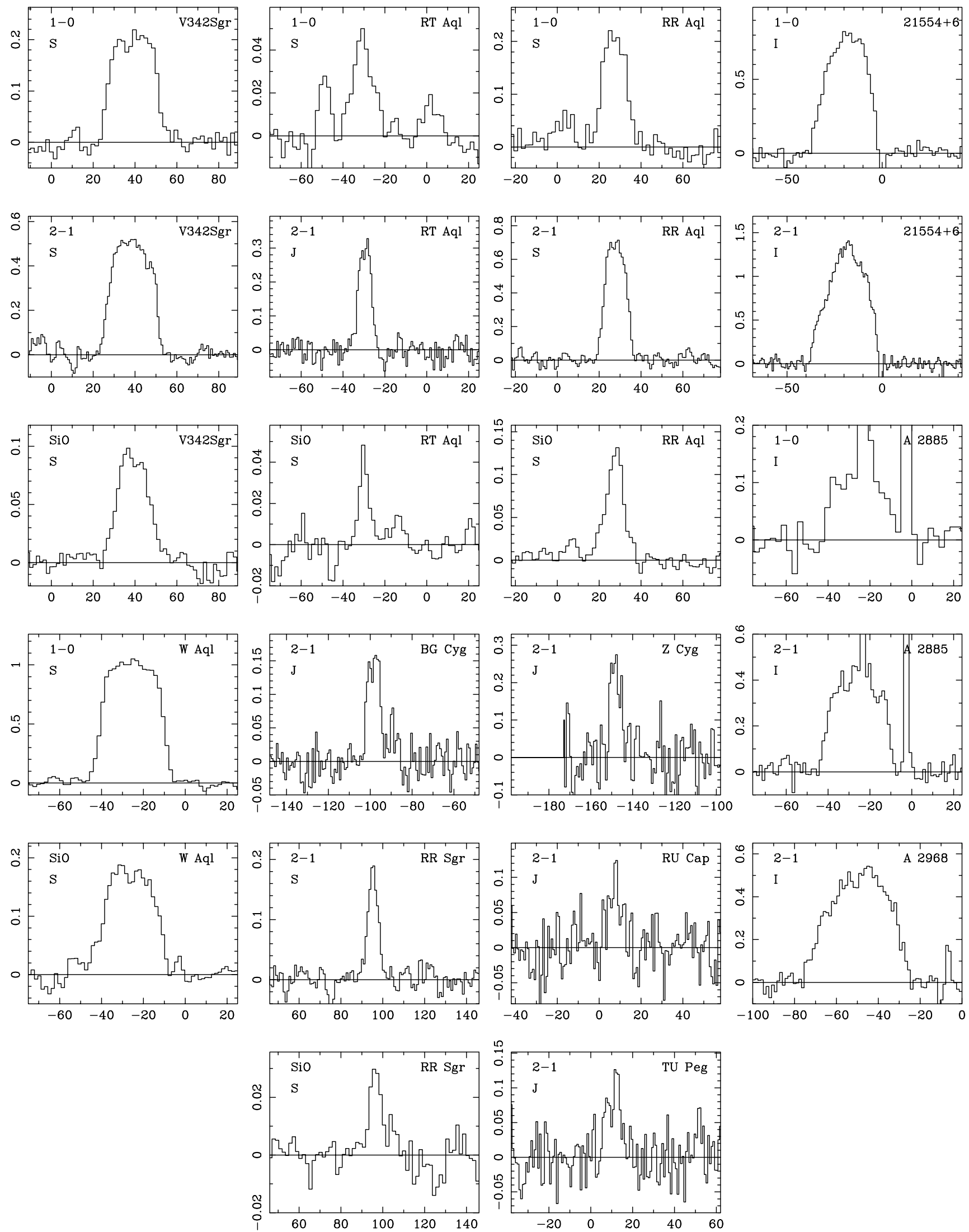

Fig. 8. As Fig. 4 
The interstellar extinction towards the sources was estimated from the model by Arenou et al. (1992), and ranges from $A_{V}=0.5-3.0$. For $\mathrm{OH} 10.1-0.1$, probably a Galactic Center source, we assumed an $A_{V}$ of 25 . Within the uncertainties, in none of the cases does the adopted value for the extinction influence the derived value for luminosity or dust mass loss rate. The model requires the expansion velocity of the outflow which is taken from the CO measurements.

The resulting fits are displayed in Figs. 10-16. We find two sources to be carbon-rich: IRAS $02408+5458$ and $06582+1507$. This was already suggested by Blommaert et al. (1993) based on the shape of the SEDs, and by Volk et al. (1993) based on the characteristic featureless LRS spectra of very red carbon stars (see also the group V sources in Groenewegen et al. 1992). This is indirectly confirmed here by the good fits obtained with amorphous carbon dust. Both stars lose mass at such high rates that any presence of silicate would result in deep silicate absorption near $10 \mu \mathrm{m}$, which is not observed. The fit to the blue part of the LRS spectrum of IRAS $02408+5458$ is not very good however. Recently, Speck et al. (1997) obtained a UKIRT CGS3 $8-13 \mu \mathrm{m}$ spectrum. Their spectrum differs in two ways from the LRS spectrum. First, their spectrum continues to rise to the blue, peaks near $9 \mu \mathrm{m}$ and only then drops in flux towards shorter wavelengths, in much better agreement with our model prediction. Second, they find strong silicon carbide absorption at $11 \mu \mathrm{m}$, which is not evident in the LRS spectrum. To simulate this, our model was calculated with $90 \%$ amorphous carbon mixed with $10 \%$ silicon carbide. The optical depth at $11.3 \mu \mathrm{m}$ in our model is 5.5. The differences between UKIRT and LRS spectrum may in part be due to the relatively poor signal-to-noise of the LRS spectrum, but the change from a nearly featureless spectrum to one with strong $\mathrm{SiC}$ absorption is real, and suggests an increase in the $\mathrm{SiC} /$ amorphous carbon ratio.

Applying the adopted kinematic distances and assuming a dust-to-gas ratio of 0.005 , we derive for IRAS 02408 +5458 a luminosity of $5700 L_{\odot}$ and a mass loss rates of $6.910^{-5} M_{\odot} \mathrm{yr}^{-1}$ and for IRAS $06582+1507$ a luminosity of $6500 L_{\odot}$ and a mass loss rate of $9.010^{-5} M_{\odot} \mathrm{yr}^{-1}$. Note that luminosity and mass loss rate scale with the adopted distance like $L \sim D^{2}$, and $\dot{M} \sim D$.

The other sources are O-rich, as evidenced by the strong silicate emission or absorption. IRAS $05284+1945$, for which no LRS spectrum is available, is an $\mathrm{OH}$ (te Lintel Hekkert et al. 1991) and $\mathrm{SiO}$ (Jiang et al. 1996) maser source. In all stars except one, a silicate dust opacity was used composed of Jones \& Merrill (1976) dirty silicate shortward of $8 \mu \mathrm{m}$, and the silicate of David \& Papoular (1990) at longer wavelengths. The exception is IRAS 18052 - 2016 (OH 10.1 - 0.1) where, based on the discussion in Suh (1999), we used $\mathrm{Mg}_{0.8} \mathrm{Fe}_{0.2} \mathrm{SiO}_{4}$ (Dorschner et al. 1995). This was the only silicate out of several species tried which resulted in at least some absorption in the
Table 4. NIR photometry

\begin{tabular}{lccc}
\hline Name & $J$ & $H$ & $K$ \\
\hline V390 Cas & $6.51 \pm 0.02$ & $5.22 \pm 0.03$ & $4.49 \pm 0.02$ \\
PT Cas & $6.85 \pm 0.02$ & $5.74 \pm 0.03$ & $5.09 \pm 0.02$ \\
AFGL 5076 & $13.9 \pm 0.3$ & $13.4 \pm 0.1$ & $9.45 \pm 0.03$ \\
Y Per & $4.63 \pm 0.02$ & $3.68 \pm 0.03$ & $3.25 \pm 0.02$ \\
GY Per & $6.39 \pm 0.02$ & $5.32 \pm 0.03$ & $4.52 \pm 0.02$ \\
C1698 & $7.66 \pm 0.02$ & $6.51 \pm 0.03$ & $5.78 \pm 0.02$ \\
U Ari & $2.08 \pm 0.02$ & $1.20 \pm 0.03$ & $0.78 \pm 0.02$ \\
\hline
\end{tabular}

$18 \mu \mathrm{m}$ region. However, in view of the still not satisfactory fit of the model to the data, further NIR data is badly needed for this source to confirm if the model prediction for the assumed dust opacity holds at shorter wavelengths. The fits to the SEDs and LRS spectra are quite good, the exception being AFGL 2968 (IRAS $22480+6002)$. However, taking the kinematic distance, we derive a luminosity of $140000 L_{\odot}$, which would make it a supergiant and not an AGB star. In support, its large expansion velocity of $26.4 \mathrm{~km} \mathrm{~s}^{-1}$ and large $60 \mu \mathrm{m}$ flux to $T(1-0)$ ratio (Josselin et al. 1998) are consistent with this interpretation. In fact, Josselin et al. classified it as a M0I object based on unpublished material (Josselin et al., in preparation). This star has not been detected in $\mathrm{OH}$. This is surprising as it is both an $\mathrm{SiO}$ (Nyman et al. 1998) and a $\mathrm{H}_{2} \mathrm{O}$ master source (Han et al. 1998). Possibly the silicate dust opacity around supergiants is different from that typical for O-rich AGB stars, or the dust is more distributed in a disk-like structure. For the other O-sources we find luminosities in the range 1120 to $30000 L_{\odot}$, and mass loss rates between $5.010^{-6}$ and $6.210^{-5} M_{\odot} \mathrm{yr}^{-1}$, again assuming a dust-to-gas ratio of 0.005 . The luminosity for IRAS $06403-0138$ is quite low $\left(1120 L_{\odot}\right)$, which might indicate that the kinematic distance is an underestimate of the true distance. Note, again, that luminosity and mass loss rate scale with the adopted distance like $L \sim D^{2}$, and $\dot{M} \sim D$

\subsection{Luminosity and distance}

Luminosities and distances are derived in various ways depending on the amount of available data.

For stars with a pulsation period the luminosity can be derived from a Period-Luminosity (PL) relation. For carbon Miras we use the relation by Groenewegen \& Whitelock (1996), which was derived for stars with periods $<520$ days. This method was used for all carbon stars in the sample, except the five stars with unknown periods. The SEDs of AFGL 5076 and 5625 were fitted by Groenewegen (1995), who assumed a luminosity of $7050 L_{\odot}$. This is the mean observed for C-stars in the LMC (see e.g. Groenewegen 1999). In the cases of IRAS $02408+5458$ and $06582+1507$ we refer to the 

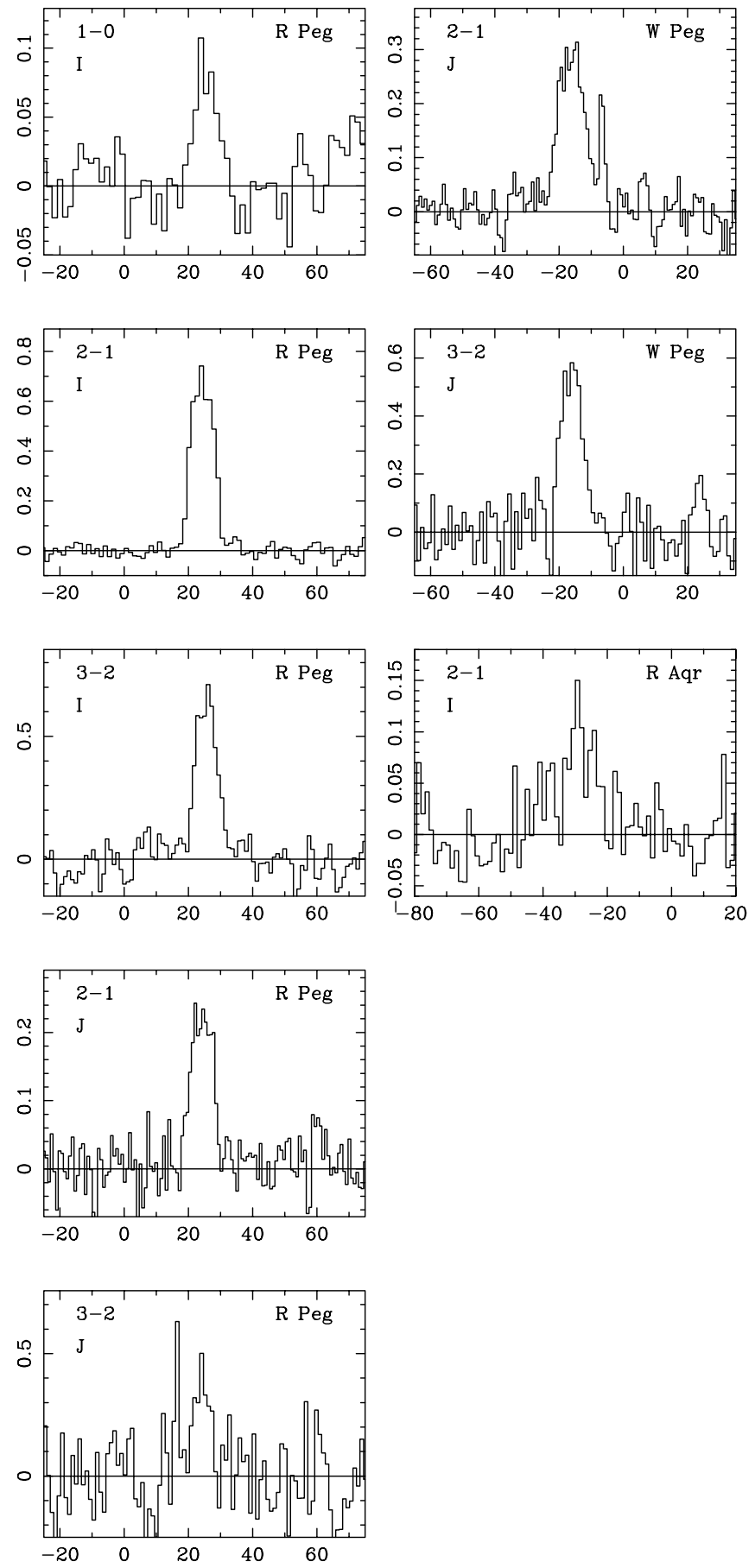

Fig. 9. As Fig. 4

previous section. C1698 is not considered any further as no sensible assumption for its luminosity and distance can be made.

For oxygen-rich Miras we use the PL-relation by Feast et al. (1989) for LMC Miras shifted by the assumed distance modulus of 18.5 (consistent with the procedure in Groenewegen \& Whitelock 1996). This was used for 49 out of the 64 O-rich stars in the sample. For the rest of the
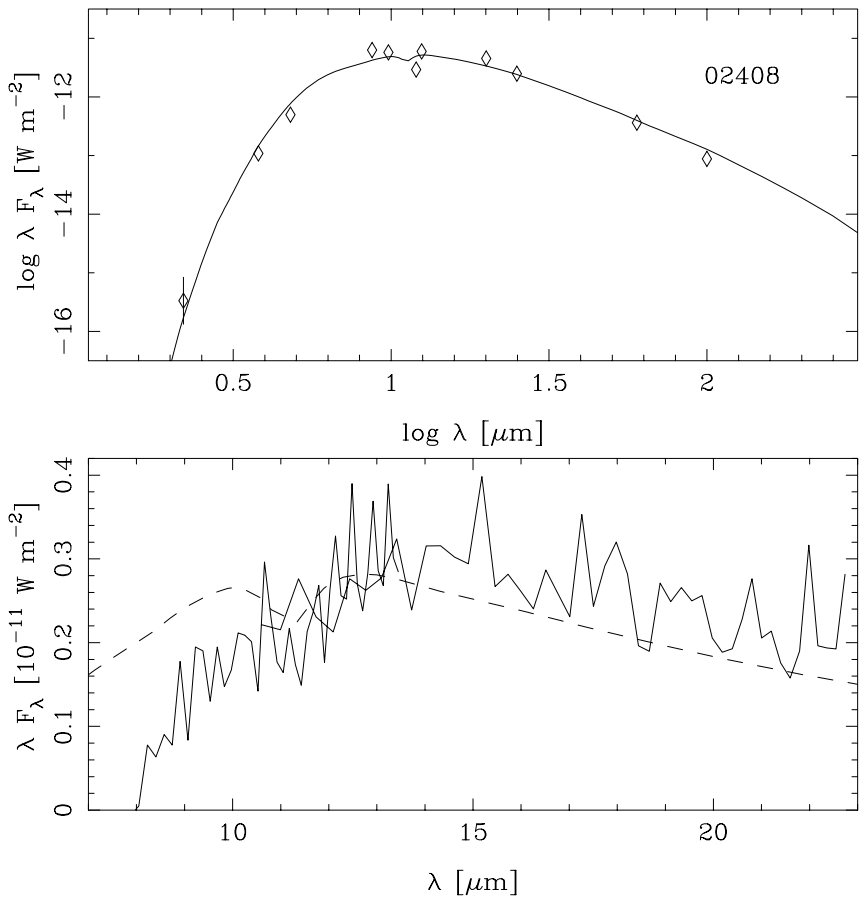

Fig. 10. Model fit to the observed SED and LRS spectrum. In the bottom panel the model is the dashed line, the LRS spectrum the solid line. This star is fitted with dust composed of $90 \%$ amorphous carbon and $10 \%$ silicon carbide
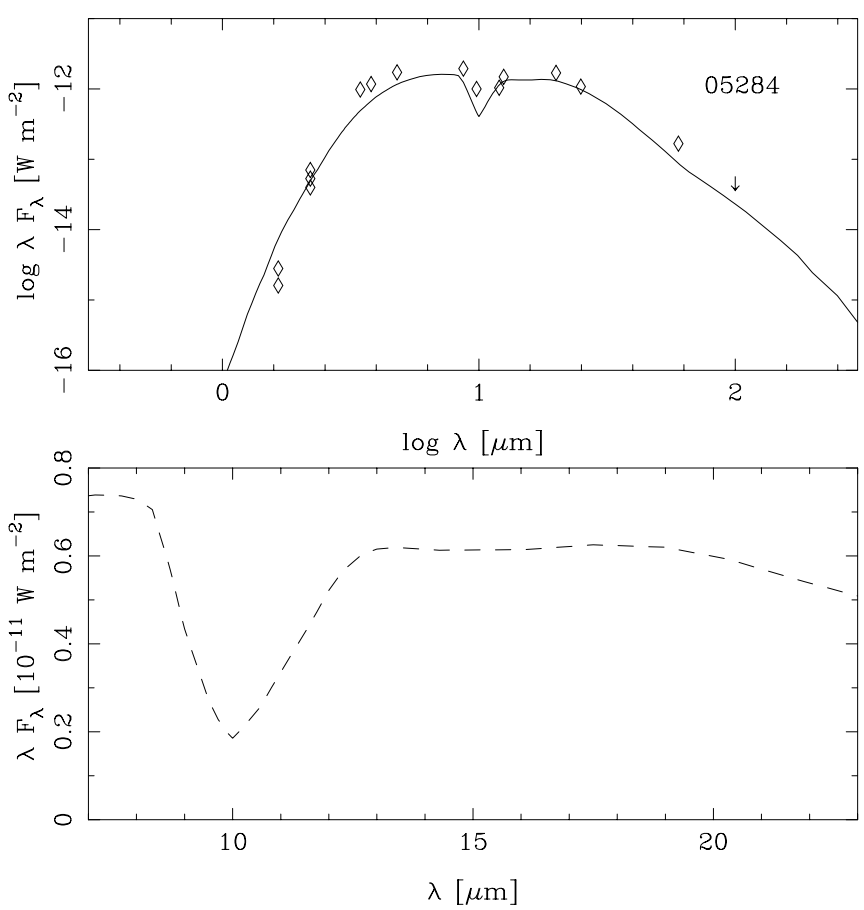

Fig. 11. As Fig. 10. Silicate dust is used 

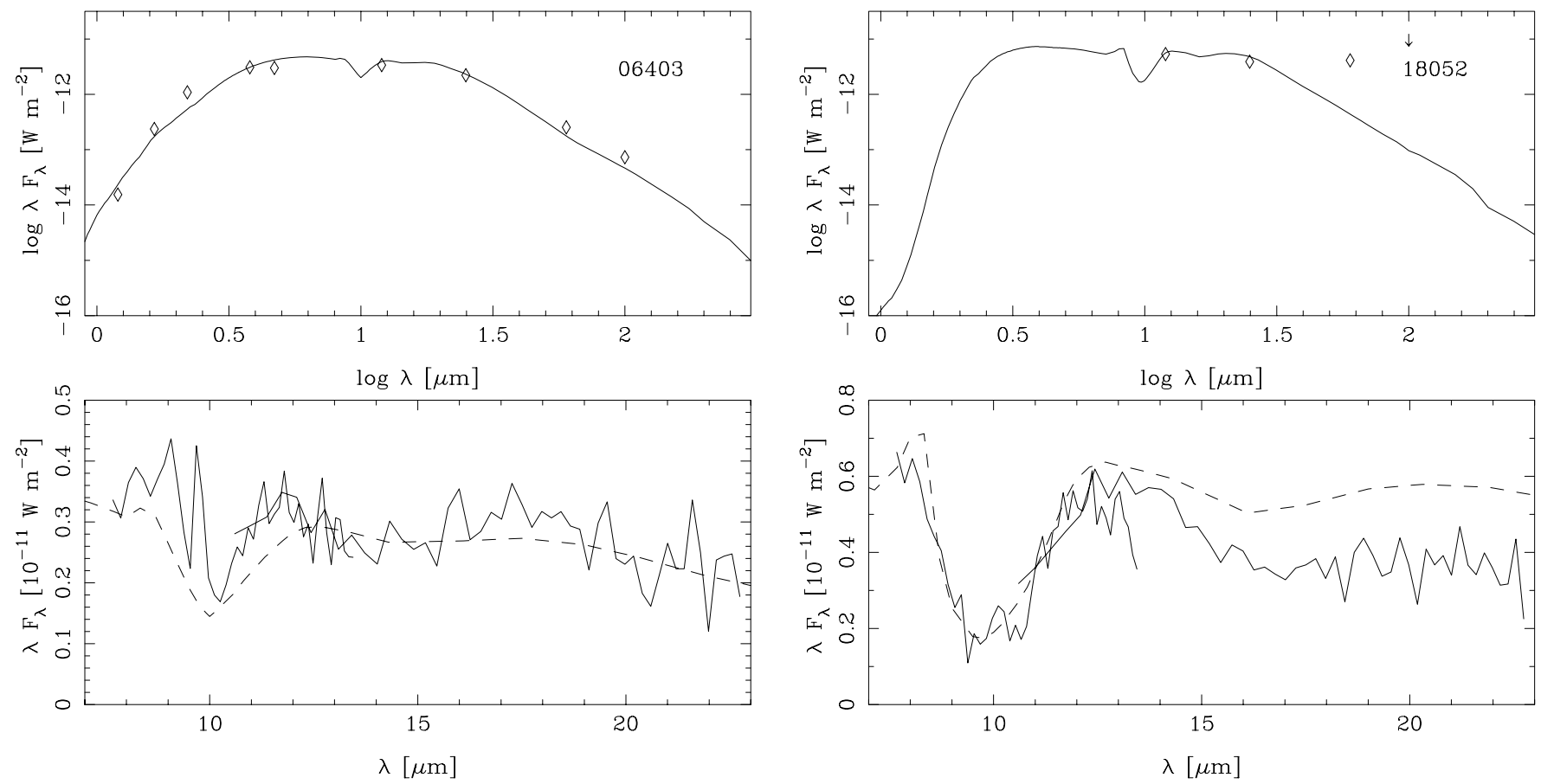

Fig. 12. As Fig. 10. Silicate dust is used

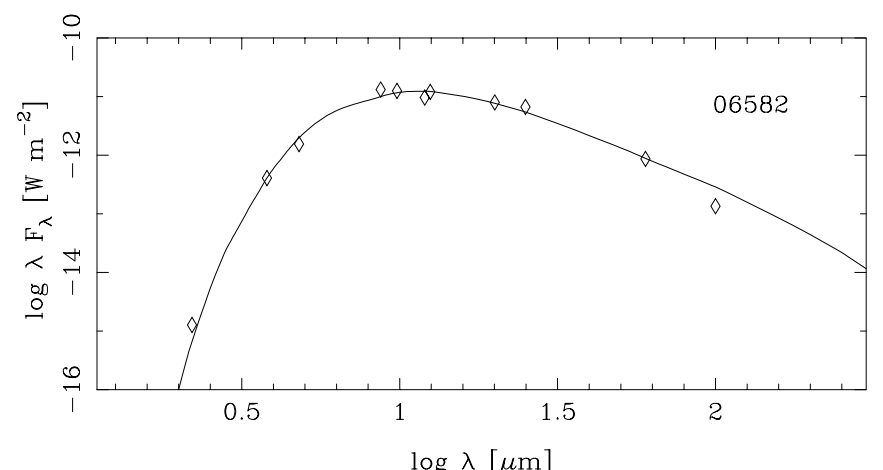

Fig. 14. As Fig. 10. Silicate dust is used
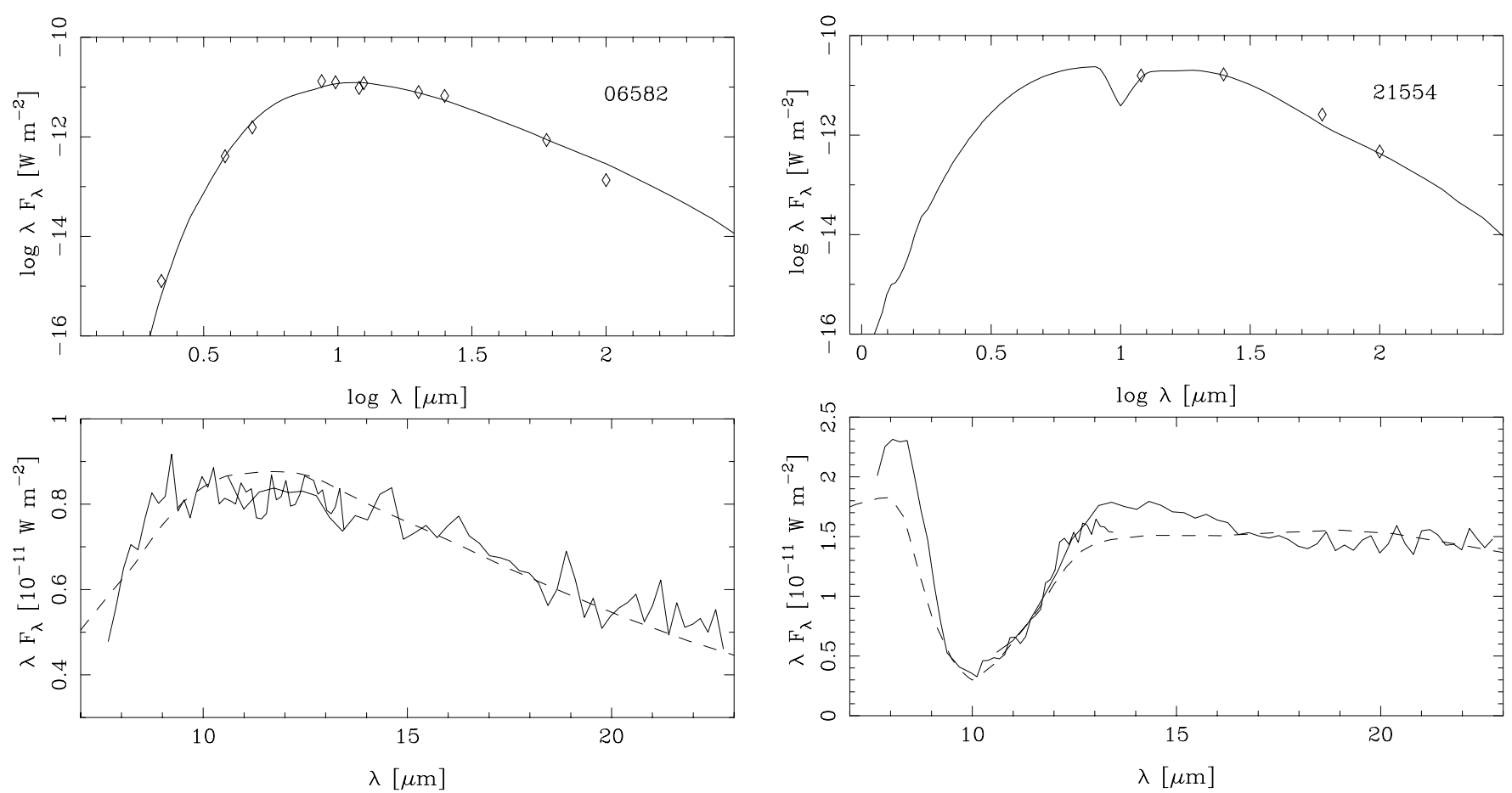

Fig. 13. As Fig. 10. Pure amorphous carbon dust is used

sample the luminosity was determined by model fitting the SED (AFGL 5093 in Groenewegen et al. 1995, and the objects discussed in Sect. 5.1), or direct integration under the SED (6 stars; Blommaert et al. 1993), with distance estimates either from kinematics (Blommaert et al. 1994), or the "phase-lag" method (van Langevelde et al. 1990). The distance and luminosity of the

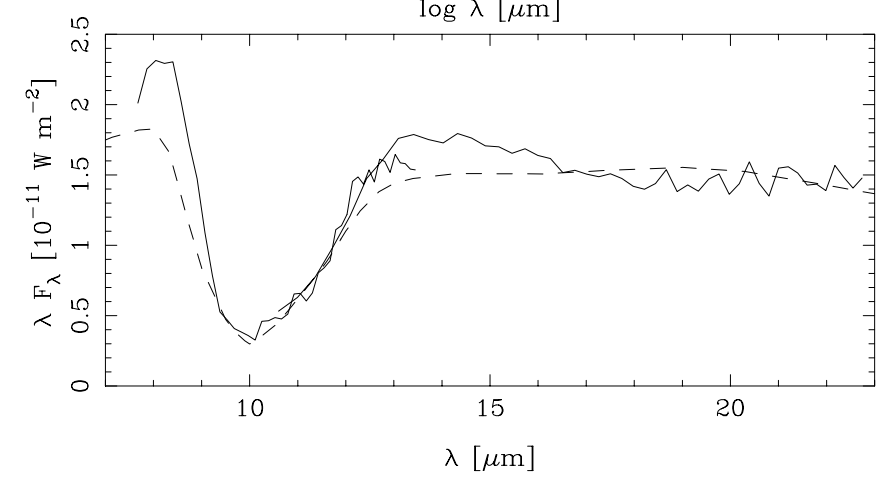

Fig. 15. As Fig. 10. Silicate dust is used

S-star W Aql is discussed in Groenewegen \& de Jong (1998) based on a comparison with S-stars in the HIPPARCOS catalog.

The SEDs of 25 out of the 35 C-stars were fitted in Groenewegen et al. (1998), two more in Groenewegen (1995), and two in Sect. 5.1 to give the distance for the adopted luminosity. For three stars, we use the Period- $M_{K}$ 

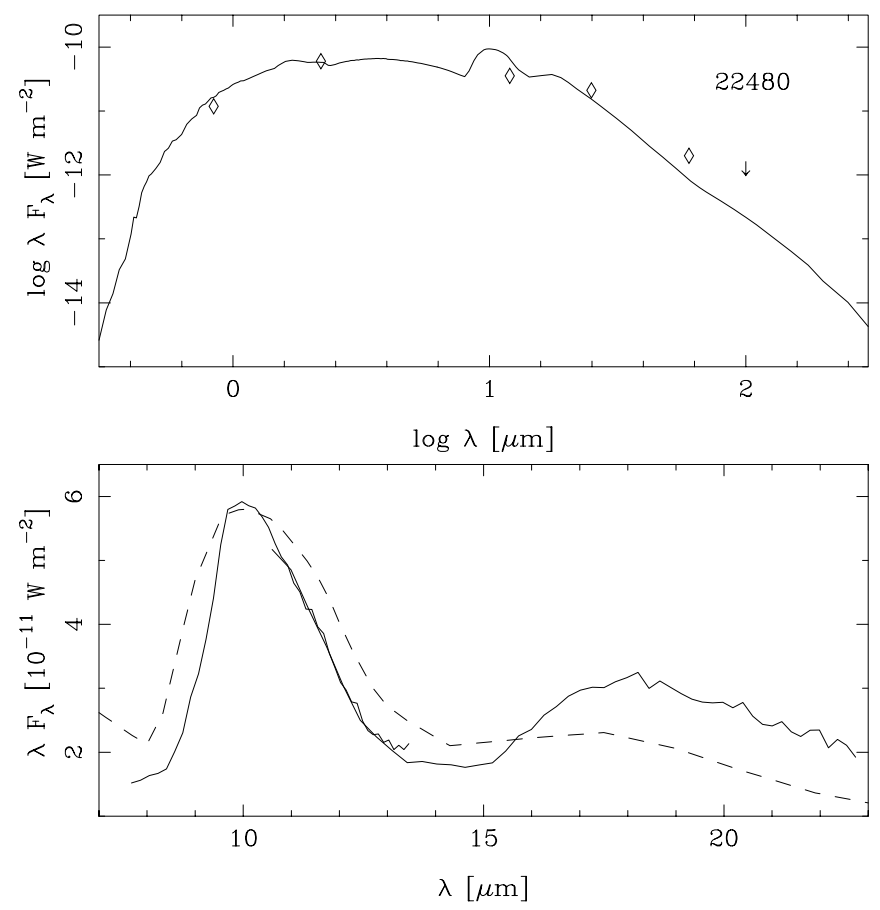

Fig. 16. As Fig. 10. Silicate dust is used

relation by Groenewegen \& Whitelock (1996), together with the observed $K$ magnitudes in Table 4, to derive the distance. For three stars no distance could be derived.

As explained above, for some of the O-rich stars there is a kinematic distance or one from the "phaselag" method. For some stars distances were derived by Whitelock et al. (1995) based on a method similar to the one described now.

For most of the stars the distance is derived from the relation between the observed $K$-band magnitude and $M_{K}$ as given by the $P-M_{K}$-relation from Feast et al. (1989). No reddening correction are applied, but they should be negligible in the $K$-band for the distances under consideration here $(\lesssim 1 \mathrm{kpc}$ for the stars this method was applied to). The observed $K$-band data were preferentially taken from the $2.2 \mu \mathrm{m}$ IRC catalog. Observations from Catchpole et al. (1979), Epchtein et al. (1985), Fouqué et al. (1992) and Kerschbaum (private comm.) were also used. No attempt was made to bring these data to a common photometric system.

For 12 out of the sample of 35 C-stars, and 33 of the 62 O-rich stars under consideration a parallax measurement in the HIPPARCOS database (ESA, 1997) is available, but only for 2 , respectively 10 , objects, has the parallax been determined with an accuracy better than $50 \%$, and only these cases have been included in Table 5 . Additionally, for $\mathrm{R}$ Leo there is the ground-based parallax determination of Gatewood (1992; $\pi=8.3 \pm 1.0$ mas) as well, which is more accurate than the HIPPARCOS parallax. One can compare the distances derived from the HIPPARCOS data and derived from the $P-M_{K}$-relation.
No infrared photometry is available for one star with a HIPPARCOS parallax. In 9 out of 11 cases, the distance based on the $P-M_{K}$-relation is larger than the HIPPARCos distance. For the stars with a poorly determined parallax $((\sigma / \pi)>0.21$, where $\sigma$ is the error in the observed parallax) this is 7 out of 8 . In one case the difference in distance is marginal, but for the other Miras it is a factor of 1.3, and then between factors of 1.9 and 4.4.

A neglect of reddening in our analysis can not be found responsible for this discrepancy. For distances $\lesssim 1 \mathrm{kpc}$, one expects visual extinctions $\lesssim 1$ magnitude, which translates into $A_{K} \lesssim 0.1 \mathrm{mag}$ (corresponding to a $\lesssim 10 \%$ shift in distance). The $P-M_{K}$-relation was applied to the Galaxy adopting a distance modulus to the LMC of 18.5. A larger distance modulus, as advocated by e.g. Feast \& Catchpole (1997), would make the galactic stars only brighter and hence would increase the discrepancy. A possibility is that the LMC is actually closer than adopted here.

A natural explanation for the discrepancy is given by the so-called Lutz-Kelker bias (Lutz \& Kelker 1973), and is related to the difference in sampled volume between a star with a parallax between $(\pi-\sigma)$ and $(\pi)$, and $(\pi)$ and $(\pi+\sigma)$ (also see Oudmaijer et al. 1998). One can introduce the concept of a "most probable parallax" (e.g. Koen 1992), and its value depends on the underlying distribution of stars and additional constrains one may have (e.g. a luminosity function). In the case of no additional information and an uniform space density the most probable parallax is $\left(\frac{1}{2} \pi\left(1+\sqrt{1-16\left(\frac{\sigma}{\pi}\right)^{2}}\right)\right.$ ) (Koen 1992). This is defined only for $(\sigma / \pi)<0.25$. Although perhaps a meaningless comparison for one star only, application of this procedure to $\mathrm{R}$ Leo would change the observed HIPPARCOS parallax of 9.87 mas to a probable parallax of 7.62 mas, and the observed ground-based parallax from 8.3 mas to a probable parallax of 7.8 mas, in very good agreement with each other. Based on the "most probable parallax" for the objects with $(\sigma / \pi)<0.25$ we adopt the following distances: $o$ Cet $(140 \mathrm{pc}), \mathrm{R}$ Car $(130 \mathrm{pc})$, and R Leo $(130 \mathrm{pc})$. For the rest of the sample of stars with Hipparcos parallaxes we prefer to use the distance based on the $P-M_{K}$-relation, because it is more reliable that the distance based on the parallax.

\subsection{The gas mass loss rate from $C O$}

Olofsson et al. (1993) give the following relation between the measured peak intensity of a $\mathrm{CO}$ line and the mass loss rate:

$\dot{M}_{\mathrm{g}}=1.4 \times \frac{T_{\mathrm{mb}} v_{\infty}^{2} D^{2} B^{2}}{210^{19} f_{\mathrm{CO}}^{0.85} s(J)}$

where $\dot{M}_{\mathrm{g}}$ is in units of solar masses per year, $T_{\mathrm{mb}}$ in Kelvin, $v_{\infty}$ in $\mathrm{km} \mathrm{s}^{-1}, D$ the distance in parsec. $B$ the FWHM size of the telescope beam in arcsec, $f_{\mathrm{CO}}$ the $\mathrm{CO}$ abundance relative to $\mathrm{H}_{2}$, and $s(J)$ is a correction factor, 
Table 5. Derived parameters

\begin{tabular}{|c|c|c|c|c|c|c|c|c|}
\hline Name & $\begin{array}{l}\text { Parallax } \\
\text { (mas) }\end{array}$ & $\begin{array}{l}\text { Lum. } \\
\left(L_{\odot}\right)\end{array}$ & $\begin{array}{l}D \\
(\mathrm{kpc})\end{array}$ & method & $\begin{array}{r}v_{\infty} \\
\left(\mathrm{km} \mathrm{s}^{-1}\right) \\
\end{array}$ & $\begin{array}{l}\dot{M}_{\text {dust }} \\
\left(M_{\odot} \mathrm{yr}^{-1}\right)\end{array}$ & $\begin{array}{l}\dot{M}_{\text {gas }} \\
\left(M_{\odot} \mathrm{yr}^{-1}\right)\end{array}$ & $\Psi$ \\
\hline V390 Cas & & 5400 & 3.10 & $4 \mathrm{~b}$ & (10) & $5.710^{-10}$ & $<5.910^{-7}$ & $<1035$ \\
\hline PT Cas & & 4400 & 5.68 & $4 \mathrm{~b}$ & (10) & 0.0 & $<2.610^{-6}$ & - \\
\hline CN Per & & 5720 & 3.09 & 1 & (10) & $1.010^{-9}$ & $<4.810^{-7}$ & $<480$ \\
\hline R For & & 5790 & 0.60 & 1 & 16.9 & $4.910^{-9}$ & $0.51-1.910^{-6}$ & 204 \\
\hline AFGL 5076 & & 7050 & 3.99 & 8 & 19.7 & $4.910^{-7}$ & $4.1-6.610^{-5}$ & 104 \\
\hline IRAS $02408+5458$ & & 5700 & 5.3 & 9 & 11.0 & $3.510^{-7}$ & $1.5-1.710^{-5}$ & 46 \\
\hline Y Per & & 3700 & 1.04 & 1 & 7.2 & 0.0 & $1.010^{-7}$ & $\infty$ \\
\hline GY Per & & 5600 & 3.25 & $4 \mathrm{~b}$ & (10) & $2.610^{-11}$ & $<6.210^{-7}$ & $<23800$ \\
\hline R Ori & & 5630 & 2.2 & 1 & 10.0 & $6.010^{-10}$ & $3.110^{-7}$ & 517 \\
\hline UV Aur & & 5800 & 0.85 & 1 & (10) & $1.010^{-9}$ & $<2.710^{-8}$ & $<27$ \\
\hline V Aur & & 5240 & 1.16 & 1 & 20.0 & $3.010^{-10}$ & $4.710^{-7}$ & 1570 \\
\hline ZZ Gem & & 4690 & 1.32 & 1 & 6.9 & $8.610^{-11}$ & $0.76-1.110^{-7}$ & 1060 \\
\hline AFGL 935 & & 7430 & 2.00 & 1 & 16.6 & $2.710^{-8}$ & $1.8-6.210^{-6}$ & 193 \\
\hline AFGL 971 & & 9910 & 1.55 & 1 & 13.4 & $4.210^{-8}$ & $0.16-1.310^{-5}$ & 193 \\
\hline CL Mon & & 7470 & 0.82 & 1 & 25.3 & $4.610^{-9}$ & $2.1-3.110^{-6}$ & 565 \\
\hline IRAS $06582+1507$ & & 6500 & 3.5 & 9 & 15.5 & $4.510^{-7}$ & $1.1-4.310^{-5}$ & 53 \\
\hline R CMi & & 5010 & 0.86 & 1 & (10) & $2.010^{-10}$ & $<6.410^{-8}$ & $<320$ \\
\hline R Vol & & 6800 & 0.75 & 1 & 19.9 & $1.110^{-8}$ & $0.8-2.410^{-6}$ & 127 \\
\hline $\begin{array}{l}\text { VX Gem } \\
\text { C1698 }\end{array}$ & & 5650 & 1.62 & 1 & $\begin{array}{l}(10) \\
(10)\end{array}$ & $8.010^{-10}$ & $<2.710^{-7}$ & $<340$ \\
\hline R Pyx & & 5420 & 1.20 & 1 & 8.8 & $8.010^{-10}$ & $1.8-2.510^{-7}$ & 263 \\
\hline V Cru & & 5600 & 1.33 & 1 & (10) & $3.010^{-10}$ & $<4.810^{-7}$ & $<1600$ \\
\hline V CrB & & 5310 & 0.71 & 1 & 7.3 & $1.410^{-9}$ & $0.49-2.910^{-7}$ & 164 \\
\hline V Oph & $3.65 \pm 1.23$ & 4390 & 0.68 & 1 & 7.8 & $9.510^{-11}$ & $3.7-6.710^{-8}$ & 526 \\
\hline AFGL 2310 & & 8720 & 0.81 & 1 & 26.0 & $7.010^{-8}$ & $5.1-7.610^{-6}$ & 97 \\
\hline AFGL 2368 & & 10280 & 0.88 & 1 & 27.5 & $8.010^{-8}$ & $0.43-1.110^{-5}$ & 71 \\
\hline R Cap & & 5120 & 1.47 & 1 & 9.6 & $1.010^{-7}$ & $1.0-8.010^{-6}$ & 27 \\
\hline AFGL 2686 & & 11440 & 1.08 & 1 & 25.2 & $1.010^{-9}$ & $0.76-1.210^{-5}$ & 910 \\
\hline V1549 Cyg & & 8030 & 1.32 & 1 & 15.5 & $2.010^{-8}$ & $4.2-6.110^{-6}$ & 275 \\
\hline AX Cep & & 5890 & 1.05 & 1 & 12.6 & $4.810^{-9}$ & $0.85-1.610^{-6}$ & 179 \\
\hline AFGL 5625 & & 7050 & 1.47 & 8 & 18.0 & $5.810^{-7}$ & $0.60-2.210^{-5}$ & 20 \\
\hline CIT 13 & & 7050 & 0.72 & 1 & 14.0 & $1.510^{-8}$ & $1.1-3.410^{-6}$ & 120 \\
\hline RZ Peg & $3.54 \pm 1.36$ & 6570 & 1.23 & 1 & 12.6 & $5.010^{-10}$ & $4.310^{-7}$ & 860 \\
\hline AO Lac & & 4400 & & 4 & (10) & $8.610^{-11} / \mathrm{kpc}^{2}$ & $<6.210^{-14} / \mathrm{pc}^{2}$ & $<720$ \\
\hline LS Cas & & 5000 & & 4 & (10) & $1.110^{-10^{\prime}} / \mathrm{kpc}^{2}$ & $<6.210^{-14} / \mathrm{pc}^{2}$ & $<560$ \\
\hline S Scl & & 6600 & 0.46 & $5 b$ & 4.5 & $1.410^{-10}$ & $<2.710^{-8}$ & $<193$ \\
\hline AFGL 230 & & 56800 & 2.9 & 2 & 12.1 & $2.510^{-7}$ & $3.1-8.910^{-6}$ & 29 \\
\hline$o$ Cet & $7.79 \pm 1.07$ & 3700 & 0.14 & $5 \mathrm{a}$ & 16.9 & $4.110^{-9}$ & $0.41-2.110^{-6}$ & 195 \\
\hline R Cet & & 2600 & 0.76 & $5 \mathrm{c}$ & 8.7 & $1.110^{-9}$ & $1.3-2.910^{-7}$ & 118 \\
\hline R Tri & & 4600 & 0.50 & $5 \mathrm{~b}$ & (10) & $2.010^{-10}$ & $<1.610^{-8}$ & $<80$ \\
\hline U Ari & & 6700 & 0.76 & $5 \mathrm{~b}$ & 4.2 & $2.810^{-10}$ & $0.19-1.210^{-7}$ & 171 \\
\hline AFGL 5093 & & 15800 & 3.2 & 7 & 10.3 & $1.210^{-7}$ & $1.7-2.510^{-6}$ & 18 \\
\hline AFGL 5097 & & 36400 & 6.5 & 3 & 12.3 & $1.410^{-7}$ & $4.6-7.210^{-6}$ & 41 \\
\hline RT Eri & & 6700 & 0.41 & $5 \mathrm{~b}$ & 7.0 & $2.910^{-10}$ & $2.6-4.110^{-8}$ & 114 \\
\hline U Hor & & 6300 & 0.94 & $5 \mathrm{~b}$ & (10) & $1.010^{-9}$ & $<2.610^{-7}$ & $<260$ \\
\hline W Eri & & 6900 & 0.93 & $5 \mathrm{~b}$ & 8.6 & $1.210^{-9}$ & $0.81-3.810^{-7}$ & 150 \\
\hline RS Eri & & 5200 & 0.64 & $5 \mathrm{~b}$ & 3.4 & $1.110^{-10}$ & $<2.610^{-8}$ & 240 \\
\hline R Tau & & 5700 & 0.59 & $5 \mathrm{~b}$ & 5.3 & $5.410^{-10}$ & $1.5-6.510^{-8}$ & 39 \\
\hline BD Eri & & 6000 & 0.90 & $5 \mathrm{~b}$ & (10) & $6.010^{-10}$ & $<4.110^{-7}$ & $<680$ \\
\hline RX Tau & & 5900 & 0.70 & $5 \mathrm{~b}$ & (10) & $6.610^{-10}$ & $<5.810^{-8}$ & $<88$ \\
\hline R Cae & & 7200 & 0.50 & $5 \mathrm{~b}$ & 8.9 & $5.710^{-10}$ & $<9.710^{-8}$ & $<170$ \\
\hline T Lep & & 6700 & 0.43 & $5 \mathrm{c}$ & 7.0 & $5.210^{-10}$ & $7.310^{-9}$ & 14 \\
\hline NV Aur & & 5600 & 1.0 & 3 & 18.3 & $5.210^{-8}$ & $0.61-1.210^{-5}$ & 152 \\
\hline U Dor & & 7300 & 0.76 & $5 c$ & 9.0 & $2.910^{-9}$ & $5.1-6.810^{-7}$ & 228 \\
\hline AFGL 712 & & 73300 & 8.0 & 3 & 13.4 & $1.410^{-7}$ & $2.3-5.210^{-5}$ & 250 \\
\hline IRAS 05284+1945 & & 2200 & 5.0 & 9 & 14.0 & $8.210^{-8}$ & $4.7-6.710^{-6}$ & 70 \\
\hline RT Lep & & 7400 & 0.92 & $5 \mathrm{~b}$ & 11.9 & $1.710^{-9}$ & $4.410^{-7}$ & 260 \\
\hline U Ori & & 6700 & 0.31 & $5 \mathrm{~b}$ & 7.9 & $7.010^{-10}$ & $0.12-2.810^{-7}$ & 123 \\
\hline IRAS 06403-0138 & & 1120 & 2.0 & 9 & 10.0 & $2.510^{-8}$ & $3.310^{-7}$ & 13 \\
\hline IRAS $07113-2747$ & & 35100 & 11.0 & 3 & 8.1 & $1.310^{-7}$ & $1.4-1.510^{-5}$ & 112 \\
\hline AS Pup & $3.95 \pm 1.02$ & 5800 & & 5 & 5.1 & $8.310^{-10} / \mathrm{kpc}^{2}$ & $<1.110^{-13} / \mathrm{pc}^{2}$ & $<133$ \\
\hline R Car & $7.84 \pm 0.83$ & 5400 & 0.13 & $5 \mathrm{a}$ & 6.8 & $6.610^{-11}$ & $<1.610^{-9}$ & 24 \\
\hline X Hya & & 5300 & 0.54 & $5 \mathrm{~b}$ & 6.4 & $2.910^{-10}$ & $2.2-4.810^{-8}$ & 110 \\
\hline R LMi & & 6800 & 0.29 & $5 \mathrm{~b}$ & 7.8 & $4.610^{-10}$ & $1.8-5.210^{-8}$ & 65 \\
\hline R Leo & $9.87 \pm 2.07$ & 5500 & 0.13 & $5 \mathrm{a}$ & 7.3 & $2.010^{-10}$ & $0.35-1.910^{-8}$ & 50 \\
\hline W Vel & $3.56 \pm 1.69$ & 7300 & 0.55 & $5 \mathrm{~b}$ & 6.4 & $4.610^{-10}$ & $<2.310^{-8}$ & $<50$ \\
\hline RZ Mus & & 6000 & 1.11 & $5 \mathrm{~b}$ & 17.2 & $3.810^{-9}$ & $0.78-2.610^{-6}$ & 360 \\
\hline AQ Cen & & 7100 & 0.86 & $5 \mathrm{~b}$ & 10.0 & $2.410^{-9}$ & $3.210^{-7}$ & 133 \\
\hline
\end{tabular}


Table 5. continued

\begin{tabular}{|c|c|c|c|c|c|c|c|c|}
\hline Name & $\begin{array}{l}\text { Parallax } \\
\text { (mas) }\end{array}$ & $\begin{array}{l}\text { Lum. } \\
\left(L_{\odot}\right)\end{array}$ & $\begin{array}{l}D \\
(\mathrm{kpc})\end{array}$ & method & $\begin{array}{r}v_{\infty} \\
\left(\mathrm{km} \mathrm{s}^{-1}\right)\end{array}$ & $\begin{array}{l}\dot{M}_{\text {dust }} \\
\left(M_{\odot} \mathrm{yr}^{-1}\right)\end{array}$ & $\begin{array}{l}\dot{M}_{\text {gas }} \\
\left(M_{\odot} \mathrm{yr}^{-1}\right)\end{array}$ & $\Psi$ \\
\hline RU Hya & & 5900 & 0.73 & $5 \mathrm{~b}$ & 7.4 & $9.710^{-10}$ & $1.310^{-7}$ & 134 \\
\hline RS Vir & & 6400 & 0.74 & $5 \mathrm{~b}$ & 6.2 & $1.510^{-9}$ & $0.99-3.810^{-7}$ & 127 \\
\hline Y Lup & & 7300 & 0.67 & $5 \mathrm{~b}$ & 12.6 & $1.410^{-9}$ & $1.510^{-7}$ & 107 \\
\hline S Ser & $3.70 \pm 1.62$ & 6800 & 0.79 & $5 \mathrm{~b}$ & 8.8 & $8.610^{-10}$ & $<2.210^{-7}$ & $<256$ \\
\hline RS Lib & & 3600 & 0.22 & $5 \mathrm{~b}$ & 5.0 & $8.210^{-11}$ & $1.810^{-8}$ & 220 \\
\hline BG Ser & & 2200 & 0.24 & 5 & 11.0 & $2.010^{-10}$ & $<2.810^{-6}$ & $<14400$ \\
\hline R Ser & $3.58 \pm 1.51$ & 6500 & 0.37 & $5 b$ & 5.8 & $2.110^{-10}$ & $0.14-1.010^{-7}$ & 330 \\
\hline RR Sco & $2.84 \pm 1.30$ & 4900 & 0.28 & $5 \mathrm{~b}$ & 4.2 & $1.010^{-10}$ & $1.110^{-8}$ & 110 \\
\hline RW Sco & & 7200 & 0.70 & $5 \mathrm{~b}$ & 10.7 & $2.410^{-10}$ & $2.1-2.210^{-7}$ & 875 \\
\hline V545 Oph & & 3700 & & 5 & 9.3 & $1.910^{-9} / \mathrm{kpc}^{2}$ & $2.110^{-13} / \mathrm{pc}^{2}$ & 111 \\
\hline V438 Sco & & 7200 & 0.87 & $5 \mathrm{~b}$ & (10) & $8.310^{-10}$ & $<1.610^{-7}$ & 193 \\
\hline WY Her & & 6900 & 1.53 & $5 \mathrm{~b}$ & 10.8 & $4.510^{-9}$ & $1.110^{-6}$ & 244 \\
\hline OH $10.1-0.1$ & & 30000 & 8.0 & 9 & 27.3 & $3.110^{-7}$ & $<6.010^{-5}$ & $<194$ \\
\hline X Oph & & 5900 & 0.23 & $5 \mathrm{~b}$ & 6.3 & $1.710^{-10}$ & $1.6-1.710^{-8}$ & 94 \\
\hline V342 Sgr & & 6800 & 0.67 & $5 \mathrm{~b}$ & 15.1 & $8.010^{-9}$ & $1.5-2.410^{-6}$ & 250 \\
\hline W Aql & & 10380 & 0.68 & 6 & 18.0 & $2.110^{-8}$ & $0.68-2.410^{-5}$ & 571 \\
\hline RT Aql & & 5800 & 0.67 & $5 \mathrm{~b}$ & 6.6 & $6.510^{-10}$ & $0.83-2.110^{-7}$ & 277 \\
\hline BG Cyg & $3.96 \pm 1.41$ & 7900 & 0.77 & $5 b$ & 5.0 & $2.110^{-10}$ & $7.410^{-8}$ & 350 \\
\hline RR Sgr & & 6000 & 0.51 & $5 \mathrm{~b}$ & 5.4 & $2.810^{-10}$ & $5.410^{-8}$ & 193 \\
\hline RR Agl & & 7300 & 0.55 & $5 \mathrm{~b}$ & 8.5 & $2.110^{-9}$ & $2.1-6.010^{-7}$ & 185 \\
\hline Z Cyg & & 4500 & 0.94 & $5 \mathrm{~b}$ & 4.3 & $1.810^{-9}$ & $0.37-1.810^{-7}$ & 78 \\
\hline RU Cap & & 6300 & 1.42 & $5 \mathrm{c}$ & 7.5 & $1.910^{-9}$ & $3.810^{-7}$ & 200 \\
\hline TU Peg & & 5700 & 0.63 & $5 b$ & 8.0 & $4.310^{-10}$ & $8.410^{-8}$ & 195 \\
\hline IRAS $21554+6204$ & & 7560 & 2.5 & 9 & 16.6 & $2.510^{-7}$ & $1.0-2.810^{-5}$ & 72 \\
\hline AFGL 2885 & & 27400 & 2.30 & 2 & 16.5 & $1.410^{-7}$ & $2.2-5.710^{-6}$ & 27 \\
\hline AFGL 2968 & & 140000 & 5.0 & 9 & 26.4 & $1.410^{-7}$ & $1.4-7.210^{-5}$ & 350 \\
\hline R Peg & & 6900 & 0.46 & $5 \mathrm{~b}$ & $\begin{array}{r}6.3 \\
6.3\end{array}$ & $3.710^{-10}$ & $1.3-8.010^{-8}$ & 151 \\
\hline W Peg & $3.46 \pm 1.38$ & 6200 & 0.31 & $5 \mathrm{~b}$ & 8.0 & $3.010^{-10}$ & $6.1-7.610^{-8}$ & 253 \\
\hline R Aqr & & 7100 & 0.23 & $5 \mathrm{~b}$ & 16.7 & $1.110^{-9}$ & $8.710^{-9}$ & 7.9 \\
\hline
\end{tabular}

Methods:

1) Luminosity from the C-star PL-relation of Groenewegen \& Whitelock (1996). Distance obtained from detailed modelling of the SED (Groenewegen et al. 1998).

2) Distance from the "phase-lag" method (van Langevelde et al. 1990), luminosity from Blommaert et al. (1994), based on integration under the SED.

3) Distance from kinematics (Blommaert et al. 1994) and luminosity by the same authors, based on integration under the SED.

4) Luminosity from the C-stars PL-relation of Groenewegen \& Whitelock (1996); 4b) distance from the observed $K$-band magnitude and a Period- $M_{K}$ relation (Groenewegen \& Whitelock 1996)

5) Luminosity from the PL-relation (Feast et al. 1989) of O-rich Miras in the LMC for a distance modulus of 18.5. Distance from: (5a) most probable parallax (see text); (5b) from the observed $K$-band magnitude and a Period- $M_{K}$ relation (Feast et al. 1989); (5c) as quoted by Whitelock et al. (1995).

6) From Groenewegen \& de Jong (1998).

7) Distance from the "phase-lag" method (van Langevelde et al. 1990), luminosity scaled from Groenewegen et al. (1995) based on model fitting of the SED. The dust mass loss rate is also derived from the model fitting, appropriately scaled from Groenewegen et al. (1995).

8) Luminosity adopted, distance and dust mass loss rate from model fitting to the SED (Groenewegen 1995), scaled to the adopted velocity. 9) Distance from kinematics (or adopted for IRAS 05285+1945; OH 10.1-0.1 assumed to be near the galactic center at $8.0 \mathrm{kpc}$ ), luminosity and dust mass loss from model fitting of the SED (this paper, Sect. 5.1).

equal to unity for the $J=1-0$ transition and 0.6 for the $J=2-1$ transition.

Equation (1) was calibrated for $J=2-1$ and $1-0$ transition but in view of the increasing number of available $J=3-2$ observations, determination of $s(3)$ would be useful. This was done on the basis of (rewriting Eq. (1) for given stellar parameters):

$s(3)=s(2)\left(\frac{T_{3-2}}{T_{2-1}}\right)\left(\frac{B_{3-2}}{B_{2-1}}\right)^{2}$

and only simultaneous observations with the same telescope were used in its application. Based on three stars observed with the CSO by Knapp et al. (1998) a geometric mean of $s(3)=0.43$ is derived. Based on our own JCMT data for 7 stars we find $s(3)=0.43$, and our own IRAM data on 6 stars results in $s(3)=0.24$. The latter value is clearly off, and indicates a possible calibration error (a beam efficiency which is lower), or a contribution from the error beam. If the latter is true it should be less of a problem with the smaller JCMT and CSO telescopes. The IRAM $J=3-2$ data is not used to make an estimate of the mass loss rate, and in the other cases $s(3)=0.43$ is used.

The gas mass loss rate is derived using both our $\mathrm{CO}$ results and the $\mathrm{CO}$ data in the papers listed in Table 1. Equation (1) was used with $f_{\mathrm{CO}}=510^{-4}$ for O-rich stars and $810^{-4}$ for C-stars to calculate the mass loss rate per unit distance. The gas mass loss rate listed in Table 5 is the range between minimum and maximum value in the case of multiple determinations. 
The expansion velocity is required in this calculation and the finally adopted values are listed in Table 5. They are an average of the available $\mathrm{CO}$ data (ours and literature). When no, or only poor, CO data was available the expansion velocity is taken from $\mathrm{OH}$ and $\mathrm{SiO}$ data, when available, without any correction for the possible difference between the $\mathrm{CO}$ and $\mathrm{OH} / \mathrm{SiO}$ expansion velocity. In other cases a value of $10 \mathrm{~km} \mathrm{~s}^{-1}$ is adopted.

\subsection{The dust mass loss rate}

The dust mass loss rate can be derived from a detailed modelling of the SED. This was done for most of the carbon-rich Miras in Groenewegen et al. (1998). In those cases the values they derived from the fit (their Table 3) have been used, appropriately scaled for the slightly different velocities adopted in some cases. The same was done for AFGL 5076 and 5625 (Groenewegen 1995), and AFGL 5093 (Groenewegen et al. 1995), and the stars analysed in Sect. 5.1.

For the rest of the sample the relation between the 60 (or 25) $\mu \mathrm{m}$ flux and the dust mass loss rate is used. From Jura (1988) we have:

$\dot{M}_{\mathrm{d}}=C v_{15} D^{2} L_{4}^{-0.5} \lambda_{10}^{0.5} \kappa_{150}^{-1} F(\lambda)$

where $\dot{M}_{\mathrm{d}}$ is the dust mass loss rate in units of solar masses per year, $C$ is a constant, $v_{15}$ is the dust expansion velocity in units of $15 \mathrm{~km} \mathrm{~s}^{-1}, D$ the distance in $\mathrm{kpc}, L_{4}$ the luminosity in units of $10000 L_{\odot}, \lambda_{10}$ the wavelength in units of $10 \mu \mathrm{m}$ where the peak of the energy distribution occurs, and $\kappa_{150}$ the opacity at $60 \mu \mathrm{m}$ in units of $150 \mathrm{~cm}^{2} \mathrm{~g}^{-1} . F(\lambda)$ is the excess emission at wavelength $\lambda$ in Jansky. Jura (1988) uses $C=7.710^{-10}$ and $\lambda=$ $60 \mu \mathrm{m}$.

It is clear that the excess emission should be used in Eq. (2), but in many applications, the observed flux has been used (see the discussion in Groenewegen \& de Jong 1998). Following that paper we have corrected for the photospheric contribution to the observed flux based on a simple radiative transfer model. This correction is explained for O-rich stars in Groenewegen \& de Jong (1998; their Fig. 4). In the case of carbon stars we performed similar calculations, which are based on a dust shell composed of amorphous carbon mixed with $10 \%$ silicon carbide around a central star with a blackbody temperature of $2500 \mathrm{~K}$. The inner dust temperature was set at $1200 \mathrm{~K}$. Shells of increasing optical depth were calculated and the fraction of excess emission determined as a function of $S_{12} / S_{25}$ ratio (see Fig. 17).

The constant $C$ in Eq. (3) at $\lambda=60 \mu \mathrm{m}$ is $1.3410^{-9}$ for O-rich star models (Groenewegen \& de Jong 1998) and $5.3510^{-10}$ for C-star models. At $\lambda=25 \mu \mathrm{m}$ these numbers are $2.4310^{-10}$ and $1.4910^{-10}$, respectively ${ }^{1}$.

\footnotetext{
1 Note that the number of $7.6710^{-11}$ in Groenewegen et al. (1998) is incorrect. Since the relation at $25 \mu \mathrm{m}$ was not
}

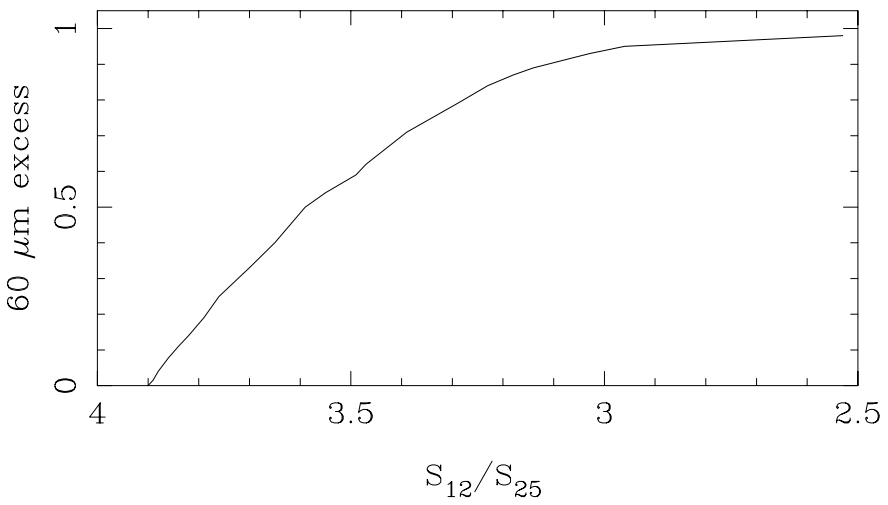

Fig. 17. Fraction of excess emission at $60 \mu \mathrm{m}$, as a function of flux-ratio at 12 and $25 \mu \mathrm{m}$, for the model described in Sect. 5.4

\section{Results of the analysis}

In this section some implications are discussed. Figure 18 shows the relation between the gas mass loss rate based on the analysis described in the previous sections, and from the combination of the dust mass loss rate derived from model fitting of the SEDs and the theory of radiation pressure on dust (Groenewegen et al. 1998) for 19 C-stars in common. The results are in perfect agreement for mass loss rates higher than $10^{-5.5} M_{\odot} \mathrm{yr}^{-1}$, and there is some indication that the mass loss rate from CO is slightly less that that derived in Groenewegen et al. (1998) for small mass loss rates. An unweighted least-squares fit gives:

$\log \dot{M}$ (previous $)=(0.85 \pm 0.09) \times \log \dot{M}($ this paper $)$

$$
-(0.77 \pm 0.55) ; \sigma=0.24 \text {. }
$$

This implies that two completely independent methods give mass loss rates that agree to better than a factor of two, and that over a wide range of mass loss rates.

Figure 19 shows the relation between mass loss rate and pulsation period for the present sample. The solid line is the relation for C-Miras derived in Groenewegen et al. (1998). It seems that at the shorter period the mass loss rates for the O-rich stars are significantly smaller than for C-stars, and they do not follow the relation for C-stars found by Groenewegen et al. (1998). Although a detailed comparison will be deferred to a future paper, we note here that some semi-regulars (SRs) of spectral type S (see Groenewegen \& de Jong 1998), and O-rich SRs (Kerschbaum et al. 1996) have mass loss rates between $10^{-8}$ and $10^{-7} M_{\odot} \mathrm{yr}^{-1}$ at periods between 100 and 250 days. A second implication of the present data is that the mass loss rate seems to level off above $P=500$ days at a level near $10^{-5} M_{\odot} \mathrm{yr}^{-1}$, independent of chemical type.

actually used this has no consequences on any of the results in that paper. 


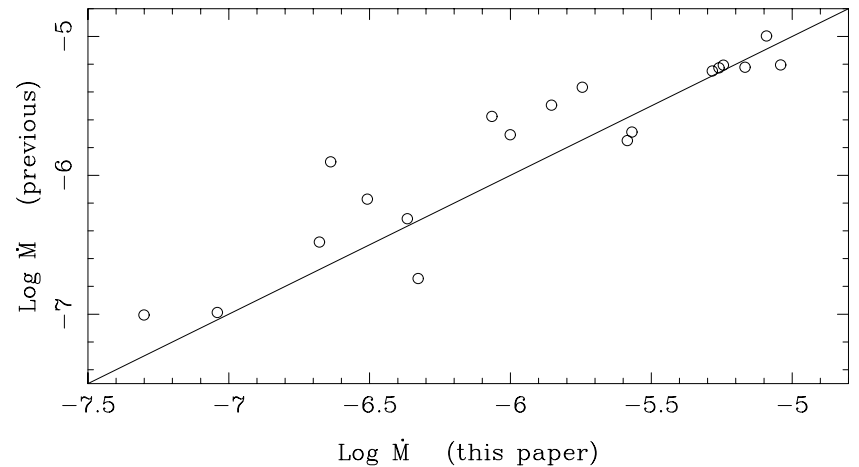

Fig. 18. Comparison between the total mass loss rates, for carbon stars only, derived in this paper (based on CO data), and in Groenewegen et al. (1998) based on the dust mass loss rate in conjunction with radiation driven wind theory. The solid line is the one-to-one relation

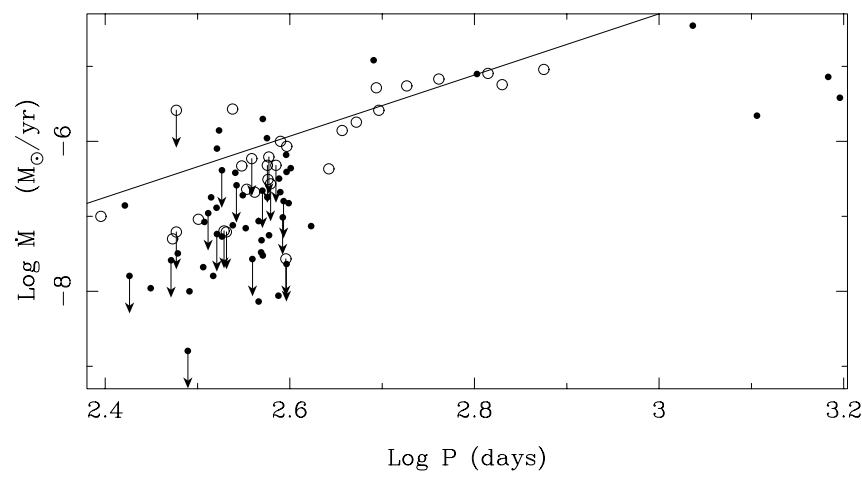

Fig. 19. The relation between $\log ($ Period) and Log (total mass loss rate). $\circ=$ Carbon stars, $\bullet=$ O-rich stars. The solid line is the relation derived in Groenewegen et al. (1998) for C-Miras

Figure 20 shows the gas-to-dust ratio as a function of pulsation period. A similar trend is observed in the gas-to dust ratio. At short periods the gas-to-dust ratio of C-stars seems to be higher than for the corresponding O-rich stars. At longer periods, there seems to be no dependence on chemical type, and the gas-to-dust ratio decreases.

Figure 21 shows the expansion velocity as a function of period. Particularly for O-rich stars an upper limit of $18 \mathrm{~km} \mathrm{~s}^{-1}$ is observed around $P=600$ days in the present sample with decreasing expansion velocities towards shorter and longer periods. Similar upper limits on the expansion velocity of O-rich stars of about $20 \mathrm{~km} \mathrm{~s}^{-1}$ can be observed in Loup et al. (1993), Groenewegen \& de Jong (1998), Kerschbaum \& Olofsson (1999). Note that some known O-rich sources in our sample have larger expansion velocities, but they are known supergiants, or no period is known. The upper limit on the expansion velocity is in qualitative agreement with the calculations presented by Habing et al. (1994) for AGB stars which predict a maximum attainable expansion velocity for a

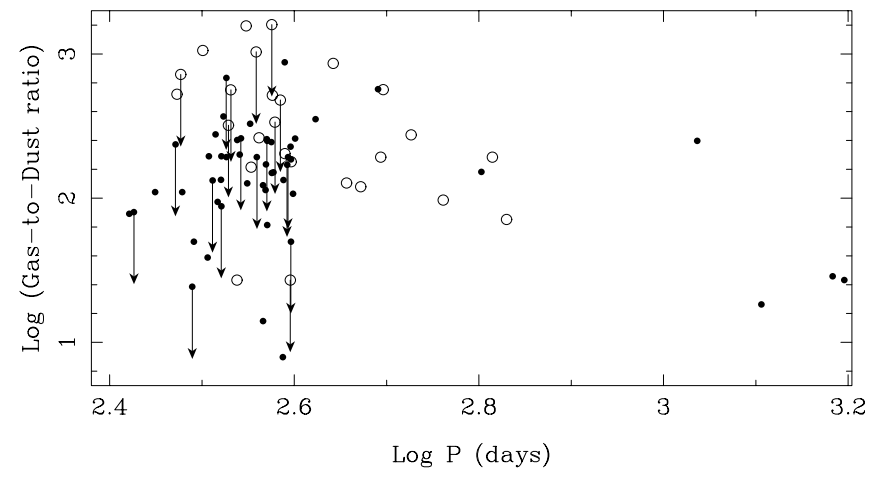

Fig. 20. The relation between Log (Period) and Log (gas-todust ratio). Symbols as in previous figure

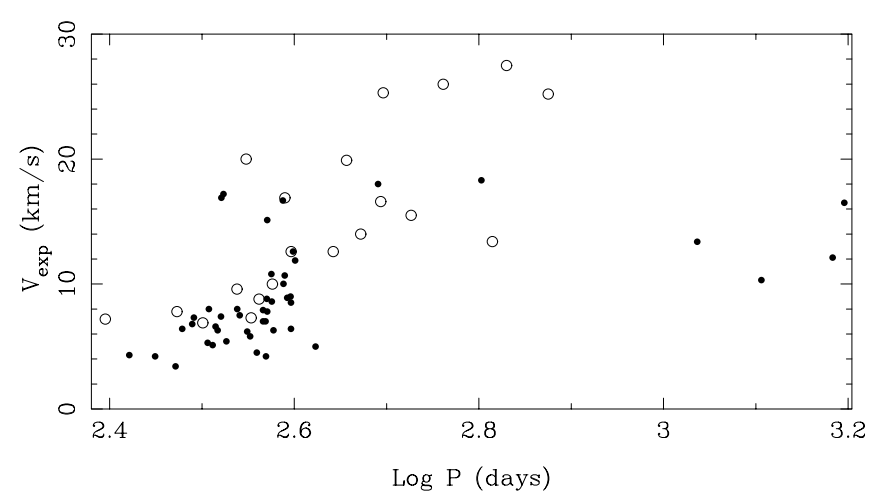

Fig. 21. The relation between Log (Period) and expansion velocity. Symbols as in previous figure

mass loss rate of about $510^{-6} M_{\odot} \mathrm{yr}^{-1}$ in O-rich stars. Their models also predict that the expansion velocity in C-stars reaches larger values, which is indeed confirmed from our data (also see Groenewegen \& de Jong 1998).

We consider the present discussion of the results as preliminary. In a future publication we will combine the CO data, and the derived mass loss rates with other recent publication on CO measurements in MS- and S-stars (Groenewegen \& de Jong 1998), Semi-Regular and Irregular variables (Kerschbaum \& Olofsson 1998, 1999), and other literature data. Using this much larger dataset it should be possible to see if the correlations presented here hold for the other categories of variable stars, and from that also gain insight into the evolutionary connection between the Carbon-, S-type and Oxygen-rich $\mathrm{Lb}, \mathrm{SR}$ and Mira variables, which is yet not possible from the present limited dataset.

Acknowledgements. We would like to thank Franz Kerschbaum (Vienna) for providing us with unpublished IR photometry of RZ Mus. This research has made use of the SIMBAD database, operated at CDS, Strasbourg, France. Felipe Mac-Aulife and Francisco Azagra (SEST staff) are thanked for doing some of the September 1996 and September 1998 SEST observations, respectively. 


\section{References}

Arenou F., Grenon M., Gomez A., 1992, A\&A 258, 104

Benson P.J., Little-Marenin I.R., 1996, ApJS 106, 579

Bieging J.H., Latter W.B., 1994, ApJ 422, 765

Bieging J.H., Knee L.B.G., Latter W.B., Olofsson H., 1999, A\&A 339,811

Blommaert J.A.D.L., van der Veen W.E.C.J., Habing H.J., 1993, A\&A 267, 39

Blommaert J.A.D.L., van Langevelde H.J., Michiels W.F.P., 1994, A\&A 287, 479

Bujarrabal V., Fuente A., Omont A., 1994, A\&A 285, 247

Catchpole R.M., Robertson B.S.C., Lloyd Evans T.H.H., et al., 1979, SAAO Circ. 1, 61

Cho S.-H., Kaifu N., Ukita N., 1996, A\&AS 115, 117

David P., Papoular R., 1990, A\&A 237, 425

Dominik C., Gail H.-P., Sedlmayr E., Winters J.M., 1990, A\&A 240,365

Dorschner J., Begemann B., Henning Th., Jager C., Mutschke H., 1995, A\&A 300, 503

Duflot M., Figon P., Meyssonnier N., 1995, A\&AS 114, 269 (used is version 6 of their catalog (March 1998), available through the CDS)

Engels D., 1994, A\&A 285, 497

Epchtein N., Matsuura O.T., Braz M.A., et al., 1985, A\&AS 61,203

ESA, "The Hipparcos and Tycho Catalogues", 1997, ESA SP1200

Feast M.W., Catchpole R.M., 1997, MNRAS 286, L1

Feast M.W., Glass I.S., Whitelock P.A., Catchpole R.M., 1989, MNRAS 241, 375

Forveille T., Guilloteau S., Lucas R., 1990, IRAM Publication

Fouqué P., Le Bertre T., Epchtein N., Guglielmo F., Kerschbaum F., 1992, A\&AS 93, 151

Garcia-Lario P., Manchado A., Pych W., Pottasch S.R., 1997, A\&AS 126, 479

Gatewood G., 1992, PASP 104, 23

Groenewegen M.A.T., 1993, Chapter 5, Ph.D. Thesis, University of Amsterdam

Groenewegen M.A.T., 1995, A\&A 293, 463

Groenewegen M.A.T., 1999, in: "IAU Symposium 191: AGB stars", Le Bertre T., Lebre A. and Waelkens C. (eds.). Astr. Soc. of the Pacific, p. 535

Groenewegen M.A.T., Baas F., de Jong T., Loup C., 1996, A\&A 306, 241

Groenewegen M.A.T., de Jong T., 1998, A\&A 337, 797

Groenewegen M.A.T., de Jong T., van der Bliek N.S., Slijkhuis S., Willems F.J., 1992, A\&A 253, 150

Groenewegen M.A.T., Smith C.H., Wood P.R., Omont A., Fujiyoshi T., 1995, ApJ 449, L119

Groenewegen M.A.T., Whitelock P.A., Smith C.H., Kerschbaum F., 1998, MNRAS 293, 18

Groenewegen M.A.T., Whitelock P.A., 1996, MNRAS 281, 1347

Habing H.J., Tignon J., Tielens A.G.G.M., 1994, A\&A 286, 523

Haikala L.K., 1990, A\&AS 85, 875
Haikala L.K., Nyman L.-Å., Forsström V., 1994, A\&AS 103, 107

Han F., Mao R.Q., Lu J., et al., 1998, A\&AS 127, 181

Jiang B.W., Deguchi S., Hu J.Y., et al., 1996, ApJS 106, 463

Jiang B.W., Deguchi S., Hu J.Y., et al., 1997, AJ 113, 1315

Jones T.J., Bryja C.O., Gehrz R.D., et al., 1990, ApJS 74, 785

Jones T.W., Merrill K.M., 1976, ApJ 209, 509

Josselin E., Loup C., Omont A., et al., 1998, A\&AS 129, 45

Jura M., 1988, ApJS 66, 33

Kerschbaum F., Olofsson H., Hron J., 1996, A\&A 311, 273

Kerschbaum F., Olofsson H., 1998, A\&A 336, 654

Kerschbaum F., Olofsson H., 1999, A\&AS 122, 167

Kholopov P.N., Samus N.N., Frolov M.S., et al., 1985, General Catalog of Variable Stars (GCVS), Nauka, Moscow

Knapp G.R., Young K., Lee E., Jorissen A., 1998, ApJS 117, 209

Koen C., 1992, MNRAS 256, 65

Le Bertre T., 1992, A\&AS 94, 377

Lewis B.M., 1997, AJ 114, 1602

Likkel L., Miao Y., 1996, AJ 112, 301

Loup C., Forveille T., Omont A., Paul J.F., 1993, A\&AS 99, 291

Lutz T.E., Kelker D.H., 1973, PASP 85, 573

Margulis M., van Blerkom D.J., Snell R.L., Kleinmann S.G., 1990, ApJ 361, 673

Neri R., Kahane C., Lucas R., Bujarrabal V., Omont A., 1998, A\&AS 130, 1

Nyman L.-A., Booth R.S., Carlström U., et al., 1992, A\&AS 93, 121

Nyman L.-A., Hall P.J., Olofsson H., 1998, A\&AS 127, 185

Olofsson H., Eriksson K., Gustafsson B., Carlström U., 1993, ApJS 87, 267

Olofsson H., Lindqvist M., Nyman L.-A., Winnberg A., 1998, A\&A 329, 1059

Oudmaijer R.D., Groenewegen M.A.T., Schrijver H., 1998, MNRAS 294, L41

Sahai R., Liechti S., 1995, A\&A 293, 198

Speck A.K., Barlow M.J., Skinner C.J., 1997, MNRAS 288, 431

Stanek K.Z., Knapp G.R., Young K., Phillips T.G., 1995, ApJS 100,169

Stephenson C.B., 1989, Pub. Warner and Swasey Obs., Vol. 3, No. 2

Suh K.-W., 1999, MNRAS 304, 389

Te Lintel Hekkert P., Caswell J.L., Habing H.J., Haynes R.F., Norris R.P., 1991, A\&AS 90, 327

van Langevelde H.J., van der Heiden R., van Schooneveld C., 1990, A\&A 239, 193

Volk K., Kwok S., Woodsworth A.W., 1993, ApJ 402, 292

Whitelock P.A., 1990, in: "Confrontation between stellar pulsation and evolution", ASP Conf. Ser. 11, Cacciari C. and Clementini G. (eds.), p. 365

Whitelock P.A., Menzies J., Feast M.W., et al., 1994, MNRAS 267, 711

Whitelock P.A., Menzies J., Feast M.W., et al., 1995, MNRAS 276, 219

Young K., 1995, ApJ 445, 872 